\title{
Stochastic Optimization Model for Coordinated Operation of Natural Gas and Electricity Networks
}

\author{
Vahid Shabazbegian ${ }^{1}$, Hossein Ameli ${ }^{1,2, *}$, Mohammad T Ameli ${ }^{1}$, Goran Strbac ${ }^{2}$ \\ 1: Department of Electrical Engineering, Shahid Beheshti University, Daneshjou Boulevard, 1983969411 , \\ Tehran, Iran \\ 2: Control and Power group, Imperial College London, SW7 2AZ, UK
}

\begin{abstract}
Renewable energy sources will anticipate significantly in the future energy system paradigm due to their low cost of operation and low pollution. Considering the renewable generation (e.g., wind) intermittency, flexible gas-fired power plants will continue to play their essential role as the main linkage of natural gas and electricity networks, and hence coordinated operation of these networks is beneficial. Furthermore, uncertainty is always found in gas demand prediction, electricity demand prediction, and output power of wind generation. Therefore, in this paper, a two-stage stochastic model for operation of natural gas and electricity networks is implemented. In order to model uncertainty in these networks, Monte Carlo simulation is applied to generate scenarios representing the uncertain parameters. Afterwards, a scenario reduction algorithm based on distances between the scenarios is applied. Stochastic and deterministic models for natural gas and electricity networks are optimized and compared considering integrated and iterative operation strategies. Furthermore, the value of flexibility options (i.e., electricity storage systems) in dealing with uncertainty is quantified. A case study is presented based on a high pressure 15-node gas system and the IEEE 24-bus reliability test system to validate the applicability of the proposed approach. The results demonstrate that applying the stochastic model of gas and electricity networks as well as considering integrated operation strategy in the presence of flexibility provides different benefits (e.g., 14\% cost savings) and enhances the system reliability in the case of contingency.
\end{abstract}

Keywords: Scheduling; Natural gas and electricity networks; Uncertainty; Two-stage stochastic programming; Monte Carlo simulation; Electricity storage systems.

Indices:

$Y \quad$ Set of terminal nodes indexed by $y(y \in Y \subseteq N)$

$N \quad$ Set of nodes indexed by $n(n \in N)$

$P \quad$ Set of pipelines indexed by $p\left(p \in P \subseteq\left(N, N^{\prime}\right)\right)$

$S \quad$ Set of scenarios indexed by $s(s \in S)$

$C \quad$ Set of compressors indexed by $c\left(c \in C \subseteq\left(N, N^{\prime}\right)\right)$

$T \quad$ Set of time indexed by $t(t \in T)$

$G \quad$ Set of thermal units indexed by $g(g \in G \subseteq B)$

$D \quad$ Set of cost function slopes indexed by $d(d \in D)$

* Corresponding author

Email addresses: v.shahbazbagian@mail.sbu.ac.ir (Vahid Shahbazbegian), h.ameli14@imperial.ac.uk (Hossein Ameli), m_ameli@sbu.ac.ir (Mohammad Taghi Ameli), g.strbac@imperial.ac.uk (Goran Strbac) 
$B \quad$ Set of busbars indexed by $b(b \in B)$

$L \quad$ Set of transmission lines indexed by $l\left(l \in L \subseteq\left(B, B^{\prime}\right)\right)$

$Q \quad$ Set of gas storages indexed by $q(q \in Q \subseteq B)$

Set of electricity storages indexed by $r(r \in R \subseteq B)$

Parameters:

$\mathrm{C}^{\text {gas }}$

$\mathrm{C}^{\mathrm{lp}}$

$\mathrm{C}^{\text {gsh }}$

$\mathcal{P}_{S}$

$D_{n . t . s}^{\text {gas }}$

Lenght

Diameter $_{p}$

$\beta_{\text {comp }}$

$\eta_{\text {comp }}$

$\mathrm{PR}^{\max }$

$Q_{c}^{\text {comp max } / \text { min }}$

$P_{c}^{\text {comp max }}$

Cost of gas supply $\left(£ / \mathrm{m}^{3}\right)$

Cost of line pack management $\left(£ / \mathrm{m}^{3}\right)$

Cost of gas load shedding $\left(€ / \mathrm{m}^{3}\right)$

Probability of each scenario (\%)

Gas demand at node $\mathrm{n}$, time $t$, and scenario $s$

Length of pipe $p(\mathrm{~m})$

$Q_{S}^{\text {sup } \max / \min }$

$\pi_{n}^{\min / \max }$

Diameter of pipe $p(\mathrm{~m})$

$Q_{p}^{\text {pipe } \min / \max }$

Gas turbine fuel rate coefficient of a compressor $\left(0.084 \mathrm{~m}^{3} / \mathrm{MJ}\right)$

$\rho^{\text {normal }}$

$\mathrm{Z}$

Overall compressor efficiency (80 \%)

Pressure ratio of compressor (1.5)

$\mathrm{R}$

$V_{p}$

$L P_{p}$

$\mathrm{T}^{\text {normal }}$

$L P_{p t}^{0}$

$C_{g}^{\text {elec }}$

$S U_{g}$

$S D_{g}$

$\mu_{\text {d.g.t }}$

VOLL

VOLW

$v_{w}$

$P t_{g}^{\min / \max }$

Maximum/minimum gas flow rate to compressor $c$

Maximum/minimum power consumption of compressor at node $c(\mathrm{~Pa})$

Maximum/minimum capacity of gas flow rate of terminal at node $y(8.5 \mathrm{mcm})$

Maximum/minimum pressures at node $n(\mathrm{~Pa})$

Maximum/minimum permitted gas flow for pipeline $p$

Gas density under standard condition $\left(0.713 \mathrm{~kg} / \mathrm{m}^{3}\right)$

Compressibility factor for natural gas $(0.95)$

Gas constant for natural gas $(518 \mathrm{~J} / \mathrm{kg} . \mathrm{K})$

Volume of gas $\left(\mathrm{m}^{3}\right)$

Linepack through pipe $p$

Gas temperature under standard condition $(288 K)$

Initial gas stored in the pipe $p$ at time $t$

Fuel cost of generation unit $g(£ / M W)$

Startup cost of generating unit $g(£)$

Shutdown cost of generating unit $g(€)$

Slope of segment $n$ pertaining to the cost function of unit $i$ at time $t(£ / M W)$

Cost of load shedding $(£ / M W)$

Cost of loss of wind $(£ / M W)$

Wind speed

$P_{d . g}^{\min / \max }$

Maximum/minimum power output of thermal unit $g$ in segment $d$ at time $t$ and scenario $s$ (MW)

$K_{g}$

$J_{g}$

$T_{g}^{\text {on }}$

$T_{g}^{\text {off }}$

$R U_{g}$

$R D_{g}$

$S U R_{g}$

$S D R_{g}$

$D_{\text {b.t.s }}^{\text {elec }}$

$B_{l}$

$\mathrm{H}_{\mathrm{v}}$

$\Psi$

$G l_{q}^{\min / \max }$

Maximum/minimum total power output of thermal unit $g$ (MW)

$Q_{q}^{\text {withdrawal max }}$ Maximum withdrawal gas of storage $q$

$Q_{q}^{\text {injection max }}$ Maximum injected gas of storage $q$

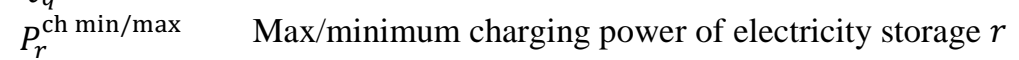

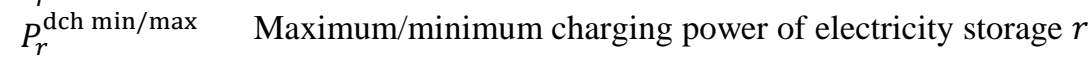

Startup cost of thermal unit $g(£)$

Shutdown cost of thermal unit $g(E)$

Minimum uptime (hour)

Minimum downtime (hour)

Ramp-up (MW/h)

Ramp-down $(\mathrm{MW} / \mathrm{h})$

ramp (MW/h)

Electricity demand at node $\mathrm{n}$, time $t$, and scenario $s$

Susceptance of line $l$

Gas heating value

Thermal efficiency of gas generator

Maximum/minimum gas level of storage $q$ 


$\begin{array}{ll}S O C_{r}^{\min / \max } & \text { Maximum/minimum state of charge of electricity storage } r \\ \eta_{\mathrm{ch}} & \text { Charging efficiency of electricity storage } \\ \eta_{\mathrm{dch}} & \text { Discharging efficiency of electricity storage }\end{array}$

\section{Variables:}

Binary decision variables:

\begin{tabular}{|c|c|}
\hline $\begin{array}{l}u_{g . t} \\
\text { Decision }\end{array}$ & $\begin{array}{l}\text { Commitment status of unit } g \text { at time } t\left(u_{g . t} \in\{0.1\}\right) \\
\text { rriables: }\end{array}$ \\
\hline$Q_{y . t . s}^{\text {sup }}$ & Gas flow rate of terminal at node $y$, time $t$, and scenario $s\left(m^{3}\right)$ \\
\hline$Q_{p . t . s}^{\text {pipe }}$ & Gas flow through pipeline $p$ at time $t$ and scenario $s\left(m^{3}\right)$ \\
\hline$G N S_{n . t . s}$ & Gas load shedding at node $n$, time $t$, and scenario $s\left(m^{3}\right)$ \\
\hline$Q_{\text {c.t.s }}^{\text {comp. }}$ & Gas flow through compressor $c$ at time $t$ and scenario $s\left(m^{3}\right)$ \\
\hline$P_{\text {c.t.s }}^{\text {comp }}$ & Consumption power of compressor $c$ at time $\mathrm{t}$ and scenario $s$ (MW) \\
\hline$\pi_{\text {n.t.s }}$ & Pressure of node $n$ at time $t$ and scenario $s$ \\
\hline$L P_{p . t . s}$ & Line pack of pipe $p$ at time $t$ and scenario $s$ \\
\hline$P g_{\text {d.g.t.s }}$ & Power output of thermal unit $g$ in segment $d$ at time $t$ and scenario $s$ (MW) \\
\hline$P t_{\text {g.t.s }}$ & Total Power output of thermal unit $g$ at time $t$ and scenario $s$ (MW) \\
\hline$E N S_{b . t . s}$ & Electrical load shedding at bus $b$, time $t$, and scenario $s$ (MW) \\
\hline$P w_{b . t . s}$ & Power output of wind generators connected to bus $b$ at time $t$ and scenario $s$ in (MW) \\
\hline$S U_{g . t}$ & Startup cost of thermal unit $g$ at time $t$ \\
\hline$S D_{g . t}$ & Shutdown cost of thermal unit $g$ at time $t$ \\
\hline$P l_{\text {l.t.s }}$ & Power flow through transmission line $l$ at time $t$ and scenario $s$ (MW) \\
\hline$\theta_{\text {l.t.s }}$ & Voltage angle at time $t$ and scenario $s$ \\
\hline$S R R_{t . s}$ & Spinning reserve requirement at time $t$ and scenario $s$ \\
\hline$Q_{\text {g.t.s }}^{\text {gen }}$ & Gas demand of thermal generator $g$ at time $t$ and scenario $s$ \\
\hline$G L_{q . t . s}$ & Gas level of storage $q$ at time $t$ and scenario $s$ \\
\hline$Q_{q . t . s}^{\text {withdrawal }}$ & Gas-withdrawal of storage $q$ at time $t$ and scenario $s$ \\
\hline$Q_{\text {q.t.s }}^{\text {injection }}$ & Gas injection into storage $q$ at time $t$ and scenario $s$ \\
\hline$P_{\text {r.t.s }}^{\text {ch }}$ & Charging power of electricity storage $r$ at time $t$ and scenario $s$ \\
\hline$P_{r . t . s}^{\text {d.t. }}$ & Discharging power of electricity storage $r$ at time $t$ and scenario $s$ \\
\hline$S O C_{\text {r.t.s }}$ & State of charge of electricity storage $r$ at time $t$ and scenario $s$ \\
\hline
\end{tabular}

\section{Introduction}

2 Climate change is among the most challenging issues in Earth, which is mainly caused due to dependency on 3 fossil fuels. Annually, a high amount of fossil fuels is consumed to generate electricity, which plays a major 4 role in producing of Greenhouse Gas (GHG) emissions [1]. Due to the urgency of the matter, a high number of 5 studies have been carried out to solve this problem, and the Paris agreement on climate change was signed between 196 countries in which employment of renewable energy resources was introduced as a part of the solution to deal with the climate change [2].

Considering the high penetration of renewable energy generation in the power system, flexible gas-fired power plants are a promising generation technology to deal with the intermittency of renewable energy resources (e.g., solar energy and wind energy). This is due to the fact that gas-fired power plants offer numerous advantages, such as (a) low cost of investment, (b) high efficiency, (c) low GHG emissions (compared to coal), and (d) flexible performance (e.g., providing short startup time and fast ramping rate) [3]. As a consequence of increasing the share of renewable energies in the power system, the imposed intermittency in the electricity network impacts the natural gas network by increasing the intermittency of the required demand for gas-fired 
power plants. Consequently, the interdependency of the operation of natural gas and electricity networks increases significantly. Therefore, the coordinated operation of these networks can be beneficial in improving reliability of the energy system and reducing the operational cost [4].

In the natural gas network, due to the low velocity of gas transportation within the network from supply points to the demand centers, a minimum level of gas is stored in the pipelines (called as linepack) to respond to sudden changes in the gas demand in time. The variability of renewable energy resources, which affects the gas demand for power generation makes linepack management more challenging [5].

Coordinated operation of natural gas and electricity networks is presented in literature through iterative and integrated strategies. In the iterative strategy, first, the operation of electricity network is optimized and the gas demand for gas-fired power plants is calculated and added to the non-electric gas demand. Then, the operation of natural gas network is optimized. If there is gas shedding, the power output of that gas-fired plants is limited accordingly, until the gas shedding equates to zero. In the integrated strategy, the operation of these networks is optimized simultaneously, and the objective function is the sum of objective functions of natural gas and electricity networks. The whole-system constraints along with a constraint, coupling these networks together are taken into account [6]-[7]. For instance, in [8], the natural gas and electricity networks were modeled, and the benefits of multi-directional compressors were examined through the iterative strategy. The obtained results indicated the benefits of each flexibility option, and electricity storage systems were presented as the optimal choice for reducing the operational costs among the studied flexibility options. Electricity storage systems are mostly charged during off-peak hours of operation period by power plants with a lower cost of operation (e.g., wind farms). These systems can be discharged during peak hours of demand, which can prevent supplying demand through expensive power plants. In addition, in the case of outage of generators, using electricity storage systems provides the possibility to supply a higher peak of demand, which enhances the reliability of the system. In [9], coordinated operation of natural gas and electricity networks was optimized considering linepack, and the impacts of natural gas network on the Unit Commitment (UC) was examined through the iterative strategy. It was demonstrated that the steady-state model of natural gas networks cannot simulate the pipelines strictly, which provides impractical solutions.

In order to study the operation of natural gas and electricity networks more realistically, uncertainties in demand and renewables should be taken into account, since the perfect foresight is not possible. For instance, the uncertainty in the electricity and gas demands is due to the randomness inherent and volatility of a high number of consumers. Furthermore, the integration of renewable energy resources entails uncertainty due to unpredictability of wind speed. Therefore, applying an approach that takes into account the uncertainty of electricity demand, non-electric gas demand, and output power of renewable energy resources, is of great importance. In [10]-[12], stochastic models of gas and electricity network were presented. In [10], an iterative strategy was applied to optimize coordinated operation of gas and electricity networks considering UC and Economic Dispatch (ED) models in the power network and non-linear equations of gas network, such as 
compressor performance and gas flow calculation. Furthermore, stochastic programming was applied to deal with the uncertainty in the output power of wind generators. The results indicated operation cost reduction of the stochastic model in comparison with the deterministic model. In [11]-[12], a number of scenarios were generated on transmission lines and generators outages, and the non-linear constraints of natural gas network were linearized using piecewise linearization. The proposed models were solved under iterative strategy, in which the obtained results illustrated the impacts of the stochastic programming and hourly demand response on the consequences of the probable outages and operation cost reduction of the system, respectively. In [13], a robust optimization model was proposed to study a coordinated operation of natural gas and electricity networks. In the proposed model, the operation cost was optimized considering the worst-case scenario (i.e., the largest possible security violation). Furthermore, to cope with complexity of the problem, alternating direction method of multipliers was applied, and electricity and gas subproblems were solved iteratively with piecewise linearized gas network constraints. Finally, the impacts of natural gas network on the UC and the benefits of employing flexible components, such as electricity storage systems, against wind generators intermittency were examined.

There are also a growing number of studies in the literature that examined the value of coordinated operation of natural gas and electricity networks through an integrated strategy [14]-[23]. A combined gas and electricity network model was developed in [14]. In this model, the linepack, gas storages, and ramp rate of gas-fired generators were considered. Finally, the obtained results demonstrated lower load shedding in the integrated networks in case of an outage of a gas terminal. Due to the dependency of Ireland gas network on gas imports from Great Britain (GB), in [15], coordinated operation of natural gas and electricity networks was optimized in both countries. Furthermore, a few scenarios were determined to study the interaction of gas and electricity networks in more detail. The results indicated that when the GB electricity system operates independently from the gas network, it is resilient against the increase of demand during peak hours of operation period. However, during coordinated operation of these networks, the ramping capability of localized generating units was limited due to the physics of gas flow and hence the reliability of the system decreased. Ability of gas storage systems to improve the operation of the power system was presented as a key finding of this study. In [16], coordinated operation of gas and electricity networks was examined through the integrated strategy, and the efficacy of flexible gas-fired plants and electricity storage systems were investigated to address electricity balancing challenges. In [17], a linearized model for coordinated operation of natural gas and electricity networks was also presented considering energy and reserve markets. In [18], sparse semidefinite programming was used to solve the similar mixed-integer nonlinear and nonconvex problem, in which analytical studies indicated the accuracy of the results. In [19], an economic dispatch model was presented for gas and electricity networks. In this study, Weymouth gas flow constraints were approximated using second-order cone relaxation. The results of this study were compared to linear models that demonstrated the acceptable accuracy of the solutions. In [20], Outer Approximation with Equality Relaxation (OA/ER) was used to solve the mixed-integer nonlinear model for coordinated operation of gas and electricity networks through integrated approach. The results of the model were compared with the optimization under iterative strategy for a gas and electricity network, which 
proved the lower cost of operation through the integrated strategy. The role of flexibility options was also investigated, and it was indicated that if the energy system is flexible enough, it is not necessary to change the current operation framework to integrated strategy.

Some studies have taken uncertainty into account in the coordinated operation of these networks through the integrated strategy of operation [21]-[23]. For instance, in [21], demand response and wind uncertainty were incorporated into the operation of natural gas and electricity networks. In order to validate the developed model, two case studies were derived, and the results proved improving the efficiency of the operation and providing profit for the decision-makers. A robust scheduling model for optimal operation of natural gas and electricity networks was presented in [22]. In this study, non-linear constraints of the natural gas network were linearized, and the model was optimized considering the worst-case scenario for electricity demand and output power of wind generators, which leads to the largest possible security violation. The results indicated the effectiveness of the model when the electricity demand and output power of renewable energy generators varied from the predicted values. In [23], a probabilistic model was proposed to optimize the operation of the gas and electricity network. In this study, Cumulant approach and Gram-Charlier expansion were applied to provide the distribution of state variables considering the effects of uncertain parameters. The results of the study showed that the applied approach can reduce the execution time and enhance accuracy.

In Table 1, the previous studies are compared in terms of optimization approach, mathematical modeling, and uncertainty consideration. By reviewing the previous studies, it is revealed that some papers have not considered the volume of natural gas in pipelines (linepack) and represented a simple model of the gas network ([11] and [12]). Some other papers have not considered electricity network constraints in detail, such as the limitation of power flow through transmission lines and constraints in UC ([13]-[15], and [17]). Furthermore, a number of studies have linearized non-linear constraints of the gas network ([9], [11]-[13], [17], [19], and [23]). Although it considerably decreases the complexity of solving the problem, provided solutions are not as strict as the non-linear model. A few studies have also considered the uncertainty of electricity demand or wind power ([8], [10], [13], [21], and [22]). However, in order to make the operational model more realistic, considering uncertainty in three vectors, including electricity demand, gas demand, and output power of wind generators, simultaneously is of great importance. 
Table 1. Systematic review of the studied papers.

\begin{tabular}{|c|c|c|c|c|c|c|c|c|c|c|c|c|c|c|c|}
\hline \multirow[b]{2}{*}{ Authors } & \multicolumn{3}{|c|}{$\begin{array}{l}\text { Electricity } \\
\text { Network }\end{array}$} & \multicolumn{2}{|c|}{$\begin{array}{l}\text { Natural Gas } \\
\text { network }\end{array}$} & \multirow[b]{2}{*}{$\begin{array}{l}\text { Modeling } \\
\text { Approach }\end{array}$} & \multicolumn{4}{|c|}{ Uncertain parameters } & \multicolumn{3}{|c|}{$\begin{array}{l}\text { Uncertainty } \\
\text { Approach }\end{array}$} & \multicolumn{2}{|c|}{$\begin{array}{c}\text { Optimization } \\
\text { approach } \\
\end{array}$} \\
\hline & 司 & ల઼ & $\begin{array}{l}n \\
己 \\
Z\end{array}$ & 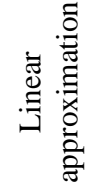 & 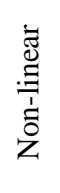 & &  & 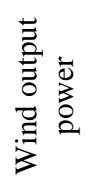 &  & 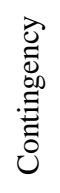 & $\begin{array}{l}0 \\
0 \\
: 0 \\
00 \\
0 \\
0 \\
0 \\
0\end{array}$ & 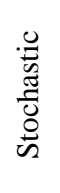 & $\begin{array}{l}\vec{v} \\
\vec{z} \\
\ddot{0}\end{array}$ & 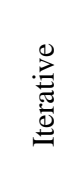 &  \\
\hline $\begin{array}{c}\text { Qadrdan et al. } \\
\text { (2017) [8] }\end{array}$ & & & $x$ & & $x$ & MINLP & & & & & & & & $x$ & \\
\hline $\begin{array}{c}\text { Liu et al. (2011) } \\
\text { [9] }\end{array}$ & & & $x$ & $x$ & & MILP & & & & & & & & $x$ & \\
\hline $\begin{array}{c}\text { Qadrdan et al } \\
\text { (2014) [10] }\end{array}$ & & & $x$ & & $x$ & MINLP & & $x$ & & & & $x$ & & $x$ & \\
\hline $\begin{array}{c}\text { Alabdulwahab } \\
\text { et al. (2017) } \\
{[11]}\end{array}$ & & & $x$ & $x$ & & MILP & & & & $x$ & & $x$ & & $x$ & \\
\hline $\begin{array}{l}\text { Zhang et al. } \\
(2016) \text { [12] }\end{array}$ & & & $x$ & $x$ & & MILP & & & & $x$ & & $x$ & & $x$ & \\
\hline $\begin{array}{c}\text { He at al. (2017) } \\
{[13]}\end{array}$ & $x$ & $x$ & & $x$ & & MILP & $x$ & $x$ & & & & & $x$ & $x$ & \\
\hline $\begin{array}{c}\text { Chuadry et al. } \\
\text { (2008) [14] }\end{array}$ & $x$ & & & & $x$ & MINLP & & & & & & & & & $x$ \\
\hline $\begin{array}{l}\text { Delwin et al. } \\
\text { (2017) [15] }\end{array}$ & $x$ & $x$ & & & $x$ & MINLP & & & & & & & & & $x$ \\
\hline $\begin{array}{l}\text { Ameli et al. } \\
\text { (2017) [16] }\end{array}$ & & & $x$ & & $x$ & MINLP & & & & & & & & $x$ & $x$ \\
\hline $\begin{array}{c}\text { Sirvent et al. } \\
\text { (2017) [17] }\end{array}$ & $x$ & $x$ & & $x$ & & MILP & & & & & & & & & $x$ \\
\hline $\begin{array}{l}\text { Menshadi et al. } \\
\text { (2017) [18] }\end{array}$ & & & & & $x$ & MINLP & & & & & & & & & $x$ \\
\hline $\begin{array}{l}\text { Sayed et al. } \\
\text { (2018) [19] }\end{array}$ & & & $x$ & $x$ & & MILP & & & & & & & & & $x$ \\
\hline $\begin{array}{l}\text { Ameli et al. } \\
\text { (2019) [20] }\end{array}$ & & & $x$ & & $x$ & MINLP & & & & & & & & $x$ & $x$ \\
\hline $\begin{array}{c}\text { Bai et al. (2016) } \\
{[21]}\end{array}$ & & & $x$ & & $x$ & MINLP & $x$ & $x$ & & & & & $x$ & & $x$ \\
\hline $\begin{array}{l}\text { Chuan et al. } \\
(2017) \text { [22] }\end{array}$ & & & $x$ & & $x$ & MINLP & $x$ & $x$ & & & & & $x$ & & $x$ \\
\hline $\begin{array}{l}\text { Yuan et al. } \\
(2017)[23]\end{array}$ & & & $x$ & $x$ & & MILP & $x$ & & $x$ & & $x$ & & & & $x$ \\
\hline This research & & & $x$ & & $x$ & MINLP & $x$ & $x$ & $x$ & & & $x$ & & $x$ & $x$ \\
\hline
\end{tabular}

(1) ED: Economic dispatch, (2) UC: Unit commitment, (3) NCUC: Network constrained unit commitment, (4) MILP: Mixed-integer linear programming, and (5) MINLP: Mixed-integer non-linear programming.

113 This paper aims to propose a stochastic model for the coordinated operation of natural gas and electricity 114 networks. For this purpose, a comprehensive and strict Mixed-Integer Nonlinear Program (MINLP) (i.e., due to 115 non-linear equations in the gas system as well as binary-variables in the generation unit commitment) 116 optimization model for the operation of natural gas and electricity networks is developed. In the proposed model, 117 constraints such as gas flow balance, gas supply limits for the terminals, linepack, pressure operational limits, 118 and gas compressor operation limits are considered. In the electricity network, a network-constrained unit commitment (NCUC) is presented, which takes into account power flow balance, spinning reserve requirements, electricity storage systems, wind generators, and characteristics of thermal generating units, such as ramp up/down, minimum uptime/downtime, and minimum/maximum generation of thermal units. In light of this, two 
122 different operational strategies, namely iterative and integrated approaches are carried out to study the 123 interaction of natural gas and electricity networks. Considering the strengths and weaknesses of the previous 124 studies, this paper fills the gap by considering the following main contributions:

- As the role of uncertainty in this problem is not deniable, Monte-Carlo simulation is applied to generate scenarios for gas demand, electricity demand, and wind power based on their Probability Density Functions (PDFs). Furthermore, a scenario reduction algorithm based on the distances between the scenarios is developed. To the best of authors' knowledge, simultaneous consideration of uncertainty in non-electric gas demand as well as electricity demand and output power of wind generators is not reported in the literature.

- A two-stage stochastic model of these networks is proposed. In the stochastic gas network operation subproblem, the optimal gas injection through terminals is obtained through the optimization in the first stage. However, the sum of costs of linepack management and gas shedding is optimized in the second stage to minimize the undesired effects of the first stage decisions. On the other hand, in the stochastic NCUC, the commitment of units is obtained through the optimization in the first stage, although the sum of costs of power generating of thermal units and load shedding is optimized in the second stage. Therefore, a stochastic model is proposed to enhance solution robustness by providing a unit commitment and amount of gas injection in which all scenarios can be met.

- Solving MINLP models are highly dependent on initial-points. Hence, an algorithm is proposed to provide initial-points and solve the MINLP model for the coordinated operation of gas and electricity networks. This algorithm consists of two main steps, which is based on solving the relaxed model in the first step, and solving the original model by adding slack variables to the gas and electricity balance equations and the corresponding penalty in the second step.

- The value of flexibility options (namely electricity storage systems) in order to deal with uncertainties in the coordinated operation strategies of gas and electricity systems is quantified. For this purpose, costs of operation, wind curtailment, and the linepack changes are investigated in the normal conditions. Furthermore, the amount of load shedding is compared with and without employing electricity storage systems in the contingency conditions (i.e., different scenarios for outages of generators).

Finally, the obtained results from the stochastic model based on reduced scenarios for uncertain parameters are compared and analyzed against a deterministic model based on the perfect foresight of the parameters during 24 hours considering the integrated and iterative strategies of operation. For this purpose, the proposed model is implemented on a 15 node gas network and the modified IEEE 24 bus reliability test system.

The structure of this study is organized as follows. After the introduction, in Section 2, the model formulation and description are presented. In Section 3, a case study is introduced to illustrate the applicability of the proposed model. Consequently, results and analyses are conducted to assess the effectiveness of the proposed model in Section 4. Finally, the conclusion is presented in Section 5. 


\section{Proposed methodology}

156 In this study, a two-stage stochastic operation model of natural gas and electricity networks is presented in 157 detail. In this model, the integrated operation strategy is compared to the iterative operation strategy with and 158 without considering uncertainty. The electricity demand, gas demand, and output power of wind generators 159 cause uncertainty in the operation of these networks. In order to solve the stochastic model for the operation of 160 natural gas and electricity networks, Monte-Carlo simulation is applied to generate scenarios representing the 161 uncertain parameters involved in the model. As it is difficult and impractical to deal with a high number of scenarios considering the physical limitation of the computers, a scenario reduction algorithm is applied. The framework for the operation of natural gas and electricity is depicted in Fig. 1.

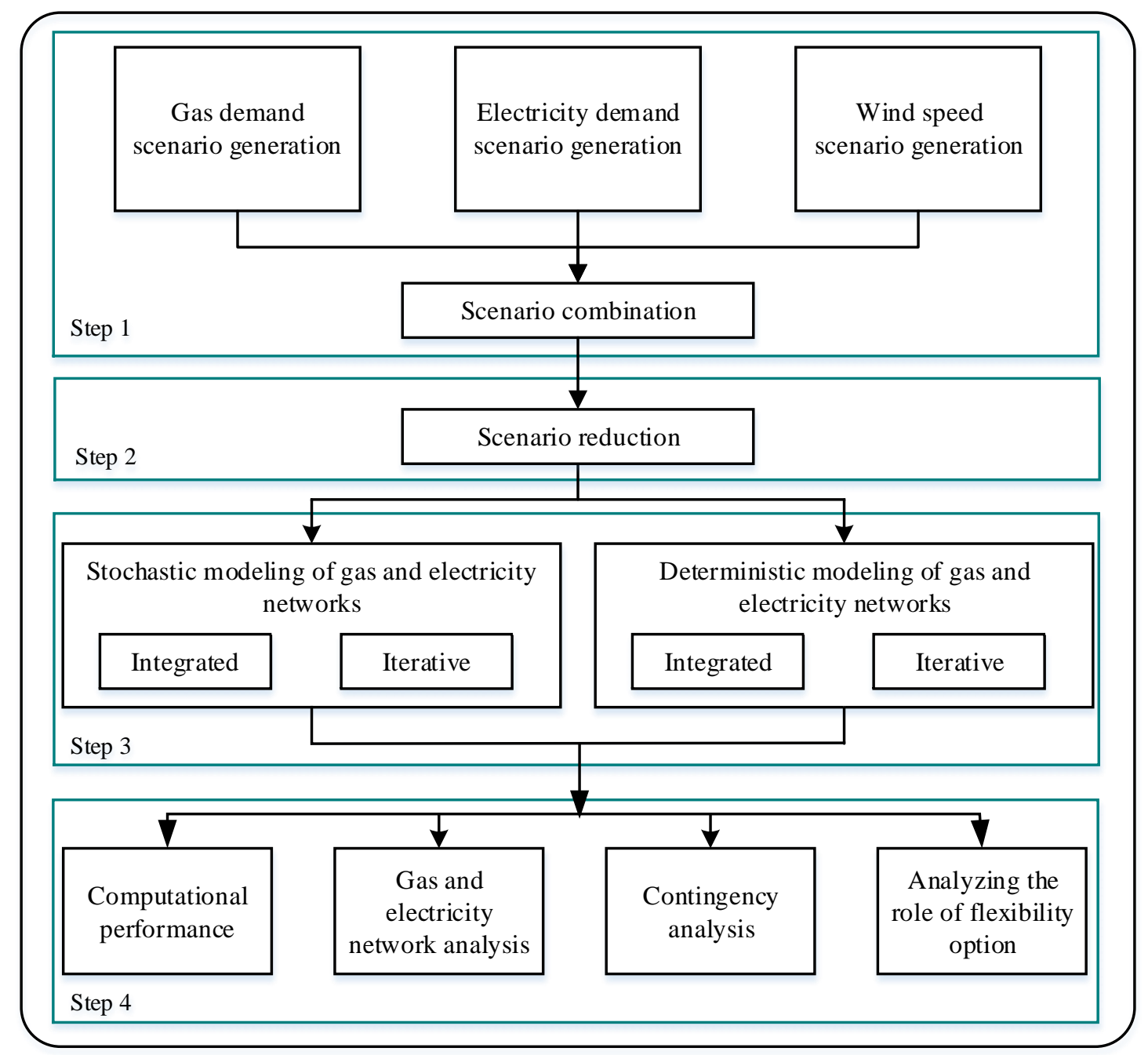

Figure 1. Proposed approach for the operation of gas and electricity networks.

\subsection{Scenario generation of uncertain gas demand, electricity demand, and wind power}

The uncertainty of gas and electricity demands are modeled using normal probability density function (PDF) as it was used in previous studies, such as [24]-[26]. Furthermore, in the previous studies, the uncertainty of wind speed was modeled using Weibull PDF [27]. The output power of wind generators is calculated according to the wind speed, cut-in speed, cut-out speed, rated speed, and rated power of turbines [24]. After scenario 
generation, the generated scenarios of each parameter are combined to form a set of scenarios. In the combined scenarios, each scenario is composed of a vector of three elements (gas demand, electricity demand, and wind power) (Equation (1)).

$$
S_{i}=\left[D_{t}^{g a s}, D_{t}^{e l e c}, P w_{t}\right]
$$

\subsection{Scenario reduction}

Dealing with a high number of scenarios increases the complexity of the model and solving time considerably. This is due to the complexity of the non-convex MINLP model of coordinated operation of natural gas and electricity networks, it is computationally impossible to solve this MINLP model for a high number of scenarios. Therefore, it is worthwhile solving the problem with a small number of scenarios which represents a reasonable approximation of the original scenarios.

Different scenario reduction techniques have been used in previous studies, such as forward selection and backward reduction [28]. In the forward scenario selection algorithm, one scenario which appropriately represents other scenarios is added through each iteration. This algorithm ends when a predetermined number of scenarios has been generated. The drawback of this algorithm is neglecting some extreme cases with a low probability of occurrence [29]. In the backward scenario reduction algorithm, on the other hand, one scenario from original scenarios is removed during each iteration based on the distance between the scenarios. Despite the simplicity of the implementation, this algorithm reduces the loss of information in comparison with other scenario reduction algorithms [30]-[31]. The steps of the backward scenario reduction algorithm are represented in Table 2. In this algorithm, initially, the occurrence probability of each scenario is the same $\left(\mathcal{P}_{\text {initial }}=1 / N_{s}\right)$.

Table 2. Scenario reduction algorithm.

\begin{tabular}{|c|c|}
\hline \multicolumn{2}{|r|}{ Loop } \\
\hline Step 1 & $\begin{array}{l}\text { - Calculating distance between scenarios. Distance between scenarios } \boldsymbol{S}_{\boldsymbol{i}} \text { and } \boldsymbol{S}_{j} \text { is equal to } \\
\boldsymbol{d}_{\boldsymbol{i} \boldsymbol{j}}=\left|\boldsymbol{S}_{\boldsymbol{i}}-\boldsymbol{S}_{j}\right|\end{array}$ \\
\hline Step 2 & $\begin{array}{l}\text { - Formulating the distance matrix } \mathcal{D} \text { which self-distances are equal to zero }\left(\boldsymbol{N}_{s} \times \boldsymbol{N}_{\boldsymbol{s}} \text { matrix }\right) \\
\text { - } \quad \text { Set } \boldsymbol{N}_{\boldsymbol{m}}=\boldsymbol{N}_{\boldsymbol{s}}\end{array}$ \\
\hline Step 3 & $\begin{array}{l}\left.\text { - Finding minimum distance in the matrix (except } \boldsymbol{d}_{\boldsymbol{i} i}\right) \text {. Two rows will contain minimum } \\
\text { values }\left(\boldsymbol{d}_{\boldsymbol{i} \boldsymbol{j}}=\boldsymbol{d}_{\boldsymbol{j} \boldsymbol{i}}\right) \text {. The rows are scenarios } \boldsymbol{x} \text { and } \boldsymbol{y} \text { with the probability of } \boldsymbol{\mathcal { P }}_{\boldsymbol{x}} \text { and } \boldsymbol{\mathcal { P }}_{\boldsymbol{y}}\end{array}$ \\
\hline Step 4 & $\begin{array}{l}\text { - If } \mathcal{P}_{\boldsymbol{x}}>\mathcal{P}_{\boldsymbol{y}}, \text { remove scenario } \boldsymbol{x} \text { and update the probability } \mathcal{P}_{\boldsymbol{x}}=\mathcal{P}_{\boldsymbol{x}}+\mathcal{P}_{\boldsymbol{y}} \\
\text { - Else if } \mathcal{P}_{\boldsymbol{y}}>\mathcal{P}_{\boldsymbol{x}} \text {, remove scenario } \boldsymbol{y} \text { and update the probability } \mathcal{P}_{\boldsymbol{y}}=\mathcal{P}_{\boldsymbol{y}}+\mathcal{P}_{\boldsymbol{x}}\end{array}$ \\
\hline Step 5 & $\begin{array}{ll}- & N_{m}=N_{m}-1 \\
- & \text { If } N_{m}>N^{*}, \text { go to the first step of the loop } \\
- & \text { Else end } \\
\end{array}$ \\
\hline
\end{tabular}

\subsection{Two-stage stochastic programming}

Two-stage stochastic programming is one of the forms of stochastic programming, which is represented mathematically in (2) [32]. In this equation, the vectors of $x \in R^{n}$ and $y \in R^{m}$ are the variables of the uncertain problem and realizations of unknown parameters are shown by $S=\left\{s_{1}, \ldots, s_{I}\right\} \subseteq R^{r}$ which $r$ is the number of uncertain parameters. 


$$
\begin{aligned}
& \text { First stage problem } \begin{cases}\min _{x} & z=\boldsymbol{c}^{\boldsymbol{T}} \cdot x+\mathbb{E}[Q(x, S)] \\
\operatorname{subject} \text { to } \quad & \boldsymbol{A} x=\boldsymbol{d} \\
& x \geq 0,\end{cases} \\
& \text { Second stage problem }\left\{\begin{array}{l}
\text { Where } \quad \mathbb{E}[Q(x, S)]=\sum_{s \in S} \boldsymbol{p}^{s} \cdot \boldsymbol{b}^{\boldsymbol{s}^{\mathbf{T}}} \cdot y^{s} \\
\boldsymbol{B}^{s} \cdot x+\boldsymbol{D}^{s} \cdot y^{s}=\boldsymbol{h}^{s}, s \in \mathcal{S}, y^{s} \geq 0, s \in S \\
\sum_{s \in S} \boldsymbol{p}^{s}=1
\end{array}\right.
\end{aligned}
$$

In the above equation, the first stage and second stage problems are assigned. In the first stage problem, $\boldsymbol{c}^{T}$ represents the cost coefficients, $\mathbb{E}[Q(x, S)]$ the expected value of the optimal solution of the second-stage problem, $\boldsymbol{A}$ the coefficients matrix, and $\boldsymbol{d}$ the right-hand side of the first stage constraints. In the second stage problem, $y$ denotes decision variable, $\boldsymbol{B}^{\boldsymbol{s}}$ transition matrix, $\boldsymbol{D}^{\boldsymbol{s}}$ cost matrix, and $\boldsymbol{h}^{\boldsymbol{s}}$ the right-hand side of the second stage constraints [33].

\subsection{Stochastic model of natural gas and electricity networks}

In this section, the two-stage stochastic model of natural gas and electricity networks is presented in detail, including the objective function and constraints.

\subsubsection{Objective function}

Equation (3), shows the objective function of the natural gas network. The first term of this objective function is the cost of gas supply and the second term is the expected cost of linepack management and gas shedding. The gas injection is considered as the first stage decision variable to supply gas demand under each scenario without changing the scheduled amount of gas injection. Equation (4) also shows the objective function of the electricity network. The first term of this objective function is the fuel costs of power generation, startup costs, and shutdown costs. The second term of this objective function is the expected cost of power generation, loss of load, and loss of wind. The commitment status of generators is considered as the first stage decision variable to supply electricity demand without changing unit commitment under each scenario. Consequently, the objective function of the coordinated operation of gas and electricity networks is presented in (5), which equates to the sum of natural gas and electricity networks' operational costs.

$$
Z_{\text {gas }}=\sum_{t} \sum_{y} \mathrm{C}^{\text {gas }} \cdot Q_{y, t}^{\text {sup }}+\mathbb{E}\left[Q_{1}(x, S)\right]
$$

where $\mathbb{E}\left[Q_{1}(x, S)\right]=\sum_{s} \mathcal{P}_{s^{*}} \sum_{t}\left(\sum_{n} \mathrm{C}^{\mathrm{lp}} \cdot \Delta L P_{n, t, s}+\sum_{n} \mathrm{C}^{\mathrm{gsh}} . G N S_{n, t, s}\right)$

$$
Z_{\text {elec }}=\sum_{t} \sum_{g} C_{g}^{\text {elec }} \cdot u_{g, t}+S U_{g}+S D_{g}+\mathbb{E}\left[Q_{2}(x, S)\right]
$$

where $\mathbb{E}\left[Q_{2}(x, S)\right]=\sum_{s} \mathcal{P}_{s} \cdot\left(\sum_{t} \sum_{g} \sum_{d}\left(\mu_{d, g, t}\right) \cdot P g_{d, g, t, s}\right.$ 
$\left.+\sum_{t} \sum_{b} V O L W \cdot\left(P w_{b, t}^{\max }-P w_{b, t, s}\right)\right)$

$Z_{\text {total }}=Z_{\text {gas }}+Z_{\text {elec }}$

211

212

213

214

\subsubsection{Natural gas network constraints}

In this subsection, the gas network constraints are presented. Limitation of gas injection is defined in (6), which is a first stage constraint in the natural gas network model. On the other hand, the second stage constraints of this network are shown in (7)-(16). Equation (7) shows the gas flow balance at each node of gas network and each period. Equation (8), is applied to simulate the compressible gas flow within the pipelines (Panhandle A equation) [34]. In natural gas network, compressors are used to boost the pressure between two nodes. Equation (9), shows the power consumption of the compressors prime-mover, which is added to the gas flow balance equation [35]. In this equation, superscripts "out" and "in" imply outlet and inlet of the compressors, respectively. Equations (10)-(12), define the operation limits of the compressors, such as pressure ratio, flow capacity, and maximum power. The pressure limits at each node and gas flow limits within the pipelines are also defined in (13) and (14). The gas storage operation limits are represented in (15)-(18).

$$
\begin{array}{ll}
Q_{y}^{\text {sup min }} \leq Q_{y, t}^{\text {sup }} \leq Q_{y}^{\text {sup max }} & \forall y \in Y, \forall t \in T \\
Q_{y, t}^{\text {sup }}-Q_{p, t, s}^{\text {pipe }}-Q_{c, t, s}^{\text {comp }}+Q_{q, t, s}^{\text {injection }}+G N S_{n, t, s}=D_{n, t, s}^{\text {gas }}+Q_{q, t, s}^{\text {witdrawal }} & \forall n \in N, \forall t \in T, \forall s \in S \\
\left(\pi_{p, t, s}^{\text {out }}\right)^{2}-\left(\pi_{p, t, s}^{\text {in }}\right)^{2}=\frac{18 \cdot 43 \text { Lenght }_{p}}{\left(\eta_{l}\right)^{2} \cdot \text { Diameter }_{p}^{4.854}} \cdot\left(Q_{p, t, s}^{\text {pipe }}\right)^{1.854} & \forall p \in P, \forall t \in T, \forall s \in S \\
P_{c, t, s}^{\text {comp }}=\frac{\beta_{\text {comp }} \cdot Q_{c, t, s}^{\text {comp }}}{\eta_{\text {comp }}^{\text {comp }}} \cdot\left[\left(\frac{\pi_{c, t, s}^{\text {out }}}{\pi_{c, t, s}^{\text {in }}}\right)^{\frac{1}{\beta_{\text {comp }}}}-1\right] & \\
1 \leq \frac{\pi_{c, t, s}^{\text {out }}}{\pi_{c, t, s}^{\text {in }}} \leq \mathrm{PR}^{\max } & \forall c \in C, \forall t \in T, \forall s \in S \\
Q_{c, t, s}^{\text {comp }} \leq Q_{c}^{\text {comp max }} & \\
P_{c, t, s}^{\text {comp }} \leq P_{c}^{\text {comp max }} & \forall c \in C, \forall t \in T, \forall s \in S \\
\pi_{n}^{\text {min }} \leq \pi_{n, t, s} \leq \pi_{n}^{\text {max }} & \forall c \in C, \forall t \in T, \forall s \in S \\
Q_{p}^{\text {pipe min }} \leq Q_{p, t, s}^{\text {pipe }} \leq Q_{p}^{\text {pipe max }} & \forall c \in C, \forall t \in T, \forall s \in S \\
G L_{q}^{\text {min }} \leq G L_{q, t, s} \leq G L_{q}^{\text {max }} & \forall n \in N, \forall t \in T, \forall s \in S \\
G L_{q, t, s}=G L_{q, t-1, s}+\left(Q_{q, t, s}^{\text {witdrawal }}-Q_{q, t, s}^{\text {injection }}\right) & \forall p \in P, \forall t \in T, \forall s \in S \\
0 \leq Q_{q, t, s}^{\text {witdrawal }} \leq Q_{q}^{\text {max withdrawal }} & \forall q \in Q, \forall t \in T, \forall s \in S \\
0 \leq Q_{q, t, s}^{\text {injection }} \leq Q_{q}^{\text {max injection }} & \forall q \in Q, \forall t \in T, \forall s \in S \\
& \forall q \in Q, \forall t \in T, \forall s \in S \\
& \forall q \in Q, \forall t \in T, \forall s \in S
\end{array}
$$

In addition to the aforementioned constraints, the linepack of the pipelines should be considered to meet the rapid changes in the gas demand. Equation (19), shows that the linepack through the pipes is proportional to the average pressure along the pipes in the steady-state condition. The inlet and outlet gas flow of a pipe are changed by supply and demand variation under dynamic conditions. Furthermore, the change of gas volume equates to the difference between inlet and outlet flow of the pipe (law of conservation of mass). Therefore, equation (19) is substituted with equation (20), which is an approximation of the dynamic situation ([16] and [20]). 


$$
\begin{array}{ll}
L P_{p}=\frac{\pi_{p}^{\text {average }} \cdot V_{p}}{\rho^{\text {normal }} \cdot Z \cdot R \cdot T^{\text {normal }}} & \forall p \in P \\
L P_{p, t, s}=L P_{p, t, s}^{0}+\sum_{0}^{t}\left(Q_{p, t, s}^{\text {pipe in }}-Q_{p, t, s}^{\text {pipe out }}\right) & \forall p \in P, \forall t \in T, \forall s \in S
\end{array}
$$

\subsubsection{Electricity network constraints}

In this subsection, electricity network constraints are represented [36]. Equations (21)-(24) define the first stage constraints of the electricity network. In (24) and (25), startup/shutdown costs are defined. In (23) and (24), the minimum uptime/downtime of thermal generating units is indicated. The second stage constraints are shown in (25)-(33). Equation (25) defines the power flow balance at each bus and each period. Equation (26), shows the power output of thermal generating units that is linearized through the piece-wise linear function. Equations (27)-(30) define the maximum/minimum stable output power of thermal generation units. The rampup/down constrains of thermal generating units are indicated in (29) and (30). In (31), power flow through transmission lines is expressed and in (32), the capacity of transmission lines is limited. The reserve requirements are also determined in (33). Equations (34)-(37) also define the electricity storage systems constraints.

$$
\begin{aligned}
& S U_{g, t} \geq K_{g} \cdot\left(u_{g, t}-u_{g, t-1}\right) \\
& S D_{g, t} \geq J_{g} \cdot\left(u_{g, t-1}-u_{g, t}\right) \\
& \sum_{t^{\prime}} u_{g, t} \geq T_{g}^{\text {on }} \cdot\left(u_{g, t}-u_{g, t-1}\right) \\
& \sum_{t^{\prime}}\left(1-u_{g, t}\right) \geq T_{g}^{\text {off }} \cdot\left(u_{g, t-1}-u_{g, t}\right) \\
& P t_{g, t, s}+P w_{b, t, s}-P l_{l, t, s}+P_{r, t, s}^{\mathrm{dch}}+E N S_{b, t, s}=D_{b . t . s}^{\text {elec }}+P_{r, t, s}^{\mathrm{ch}} \\
& P t_{g, t, s}=u_{g, t} \cdot P t_{g}^{\min }+\sum_{d} P g_{d, g, t, s} \\
& 0 \leq P g_{d, g, t, s} \leq P_{d, g}^{\max } \\
& u_{g, t} \cdot P t_{g}^{\min } \leq P t_{g, t, s} \leq u_{g, t} \cdot P t_{g}^{\max } \\
& P t_{g, t, s}-P t_{g, t-1, s} \leq R U_{g} \cdot u_{g, t-1}+S U R_{g} \cdot\left(u_{g, t}-u_{g, t-1}\right) \\
& P t_{g, t-1, s}-P t_{g, t, s} \leq R D_{g} \cdot u_{g, t}+S D R_{g} \cdot\left(u_{g, t-1}-u_{g, t}\right) \\
& P l_{l, t, s}=B_{l} \cdot\left(\theta_{l, t, s}^{\text {in }}-\theta_{l, t, s}^{\text {out }}\right) \\
& -P l_{l}^{\max } \leq P l_{l, t, s} \leq P l_{l}^{\max } \\
& \sum_{g} P t_{g}^{\max } \geq S R R T_{t, s}+\sum_{b} D_{b, t, s}^{\mathrm{elec}} \\
& S O C_{r, t, s}=S O C_{r, t-1, s}+\left(P_{r, t, s}^{\mathrm{ch}} \cdot \eta_{\mathrm{ch}}-P_{r, t, s}^{\mathrm{dch}} / \eta_{\mathrm{dch}}\right) \\
& P_{r, t, s}^{\mathrm{ch} \mathrm{min}} \leq P_{r, t, s}^{\mathrm{ch}} \leq P_{r, t, s}^{\mathrm{ch} \max } \\
& P_{r, t, s}^{\mathrm{dch} \min } \leq P_{r, t, s}^{\mathrm{dch}} \leq P_{r, t, s}^{\mathrm{dch} \max } \\
& S O C_{r}^{\min } \leq S O C_{r, t, s} \leq S O C_{r}^{\max }
\end{aligned}
$$$$
\forall g \in G, \forall t \in T
$$$$
\forall g \in G, \forall t \in T
$$$$
\forall g, t \in\left[1, T-T_{g}^{o n}+1\right]
$$$$
\forall g, t \epsilon\left[1, T-T_{g}^{o f f}+1\right]
$$$$
\forall b \in B, \forall t \in T, \forall s \in S
$$$$
\forall g \in G, \forall t \in T, \forall s \in S
$$$$
\forall d \in D, \forall g \in G, \forall t \in T, \forall s \in S
$$$$
\forall g \in G, \forall t \in T, \forall s \in S
$$$$
\forall g \in G, \forall t \in T
$$$$
\forall g \in G, \forall t \in T
$$$$
\forall l \in L, \forall t \in T, \forall s \in S
$$$$
\forall l \in L, \forall t \in T, \forall s \in S
$$$$
\forall t \in T, \forall s \in S
$$$$
\forall r \in R, \forall t \in T, \forall s \in S
$$$$
\forall r \in R, \forall t \in T, \forall s \in S
$$$$
\forall r \in R, \forall t \in T, \forall s \in S
$$ 
240 Gas-fired power plants and electricity-driven compressors couple the natural gas and electricity networks.

241 The gas consumption of these generators should be added to the gas flow balance. This value is calculated 242 considering the output power of gas-fired power plants in (38), and the gas flow balance in (7) is rewritten in 243 (39). Furthermore, the electricity consumption of electricity-driven compressors in (9) is also added to power 244 flow balance equation (40).

$$
\begin{array}{ll}
Q_{g, t, s}^{\text {gen }}=\psi \cdot \mathrm{H}_{\mathrm{v}} \cdot P t_{g, t, s} & \forall g \in G, \forall t \in T, \forall s \in S \\
Q_{y, t}^{\text {sup }}-Q_{p, t, s}^{\text {pipe }}-Q_{c, t, s}^{\text {comp }}+Q_{q, t, s}^{\text {injection }}+G N S_{n, t, s}=D_{n, t, s}^{\text {gas }}+Q_{g, t, s}^{\text {gen }}+Q_{q, t, s}^{\text {witdrawal }} & \forall n \in N, \forall t \in T, \forall s \in S \\
P t_{g, t, s}+P w_{b, t, s}-P l_{l, t, s}+P_{r, t, s}^{\text {dch }}+E N S_{b, t, s}=D_{b . t . s}^{\text {elec }}+P_{b, t, s}^{\text {comp }}+P_{r, t, s}^{\text {ch }} & \forall b \in B, \forall t \in T, \forall s \in S
\end{array}
$$

245

\subsection{Gas and electricity operational strategies}

As mentioned previously, integrated and iterative strategies are applied to model the operation of natural gas and electricity networks. In the iterative strategy, first, the operation of the electricity network is optimized (i.e., minimizing (4) subject to electricity network constraints (21)-(37). Afterwards, the gas requirement of gas-fired power plants is calculated from their output powers and added to non-electrical gas demand (38), and then, the operation of the natural gas network is optimized (i.e., minimizing (3) subject to gas network constraints (6)(18) and (20)). If there is either constraint violation or gas shedding due to the excess of gas requirements of the gas-fired power plants, the power outputs of those generators in (27) and (28) are limited until the total gas shedding equates to zero ([10], [16], and [20]). The worst-case that can happen is that the gas demand for power generation cannot be supplied and hence the output power of gas-fired power plants is limited to zero, and consequently, the electricity demand must be supplied via other generation types (e.g., coal power plants). Therefore, this can cause a considerable amount of load shedding in the electricity network, which increases the total cost of operation. The flowchart for an implementation of the proposed iterative strategy is illustrated in Fig. 2. 


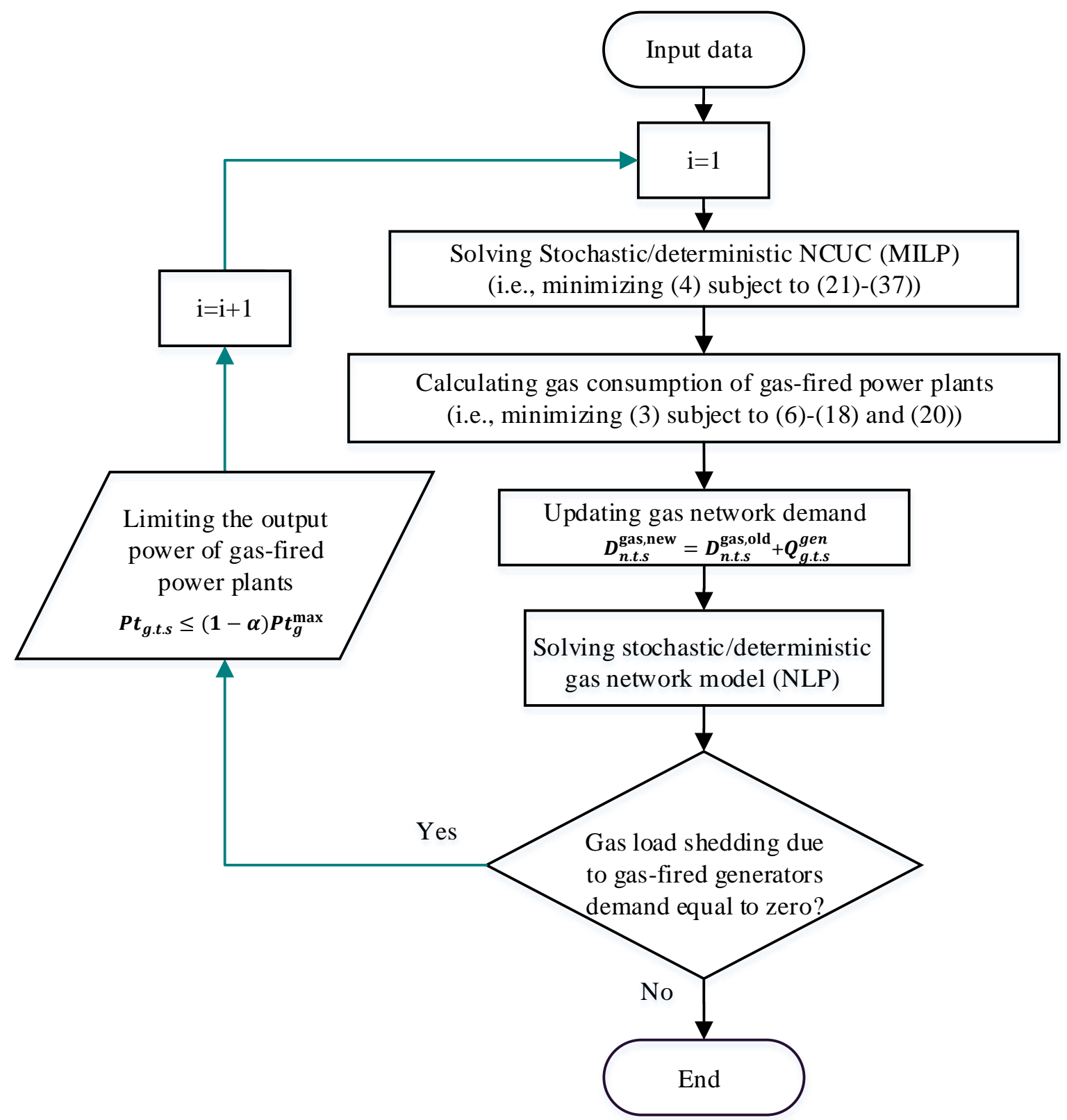

Figure 2. Structure of iterative strategy to optimize the coordinated operation of natural gas and electricity networks.

In the integrated strategy, the objective function is equal to the sum of the total operating cost of natural gas and electricity networks and all constraints are taken into account (minimizing (5) subject to (6), (8)-(18) and 262 (21)-(24), (26)-(37), and (38)-(40)). Furthermore, a constraint is considered that couples these networks (38) 263 ([13] and [19]).

The integrated model is an MINLP due to nonlinear equations in the natural gas network as well as binary variables in the unit commitment process. Therefore, solving the relaxed model that neglects integer restrictions can help to achieve optimality in the original problem [37]. The relaxed model is a nonlinear program that can provide initial-points to solve the original problem. Moreover, when the relaxed model cannot be solved, the gas or electricity subproblems should be scaled (first step) [38]. In this problem, using the obtained results from 
optimizing the relaxed model could lead to infeasibility due to the unit commitment's constraints in (21)-(24).

270 Therefore, slack variables $\left(S G_{n, t, s}\right.$ and $\left.S E_{b, t, s}\right)$ are also added in the gas and electricity balance equations (i.e., 271 substituting (39) and (40) with (41) and (42), respectively) with a considerable amount of penalty (CP) in the 272 objective function (43). Afterwards, the solution of solving the coordinated operation of gas and electricity 273 networks with the slack variables and the corresponding penalty (i.e., these variables are for optimization 274 purposes to avoid infeasibility and do not have any physical meaning) are used in an iterative manner only to 275 provide initial-points and find optimal solution. Finally, when the slack variables are equal to zero, the solution 276 is optimal. On the other hand, load shedding is used as a last action by system operators to satisfy supply277 demand balance due to lack of generation (e.g., in the case of contingency). For modeling purposes, a very high 278 penalty is set for gas and electricity load shedding ( $\mathrm{C}^{\mathrm{gsh}}$ and $\left.V O L L\right)$ to make these variables the last option to 279 maintain the supply-demand balance equation. In Fig. 3, the flowchart for solving the MINLP model through 280 the proposed algorithm is presented.

$$
\begin{array}{ll}
Q_{y, t}^{\text {sup }}-Q_{p, t, s}^{\text {pipe }}-Q_{c, t, s}^{\text {comp }}+Q_{q, t, s}^{\text {injection }}+G N S_{n, t, s} & \forall n \in N, \forall t \in T, \forall s \in S \\
\quad=D_{n, t, s}^{\text {gas }}+Q_{g, t, s}^{\text {gen }}+Q_{q, t, s}^{\text {witdrawal }}+S G_{n, t, s} & \\
P t_{g, t, s}+P w_{b, t, s}-P l_{l, t, s}+E N S_{b, t, s}=P_{b, t, s}^{\text {comp }}+S E_{b, t, s} & \forall b \in B, \forall t \in T, \forall s \in S \\
Z_{\text {total }}=Z_{\text {gas }}+Z_{\text {elec }}+\text { CP. }\left(S G_{n, t, s}+S E_{b, t, s}\right) &
\end{array}
$$




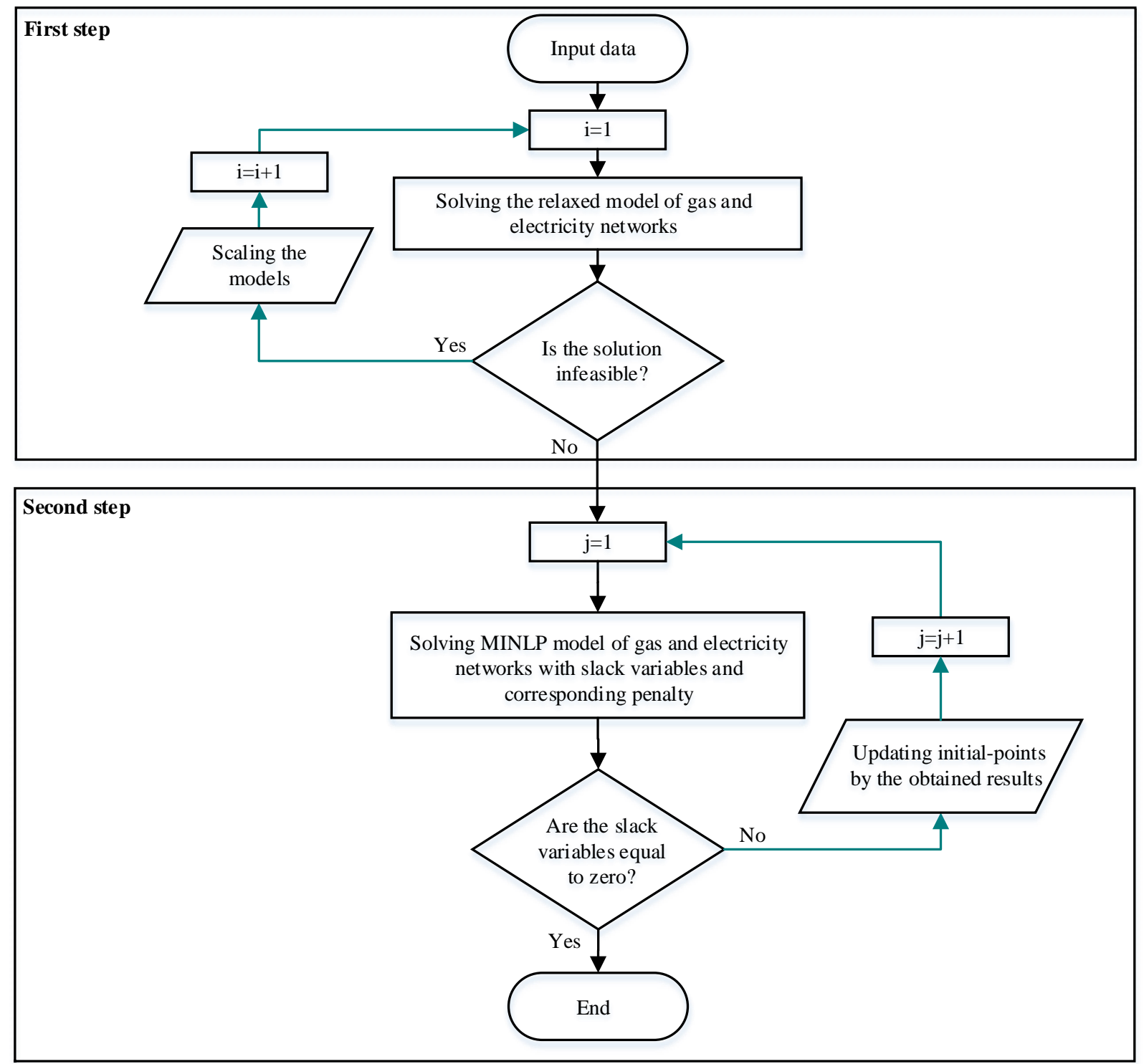

Figure 3. Proposed solving method of integrated natural gas and electricity networks model.

\section{Case study}

281 In this section, in order to validate the proposed methodology, a case study is presented (Fig. 4), and the stochastic and deterministic models of natural gas and electricity networks are optimized through integrated and iterative operational strategies (i.e., stochastic-integrated, stochastic-iterative, deterministic-integrated, and deterministic-iterative). Furthermore, a number of scenarios are determined to examine the consequence of outage of generators under different modeling (i.e., stochastic and deterministic models) and different strategies of operations (i.e., iterative and integrated strategies). Besides, the value of electricity storage systems is investigated under normal and contingency conditions to quantify the benefits of this flexibility option to deal with uncertainties.

\subsection{Natural gas network description}

The case study consists of a high pressure 15 node gas system ([39]-[40]) and the IEEE 24-bus reliability test system ([41]-[42]). The gas network consists of one gas terminal, one gas-driven compressor, two gas 
storage facilities, 15 nodes, and 16 pipelines. In the deterministic model, a perfect foresight of gas demand during 24 hours is considered in the optimization. In the stochastic model, however, the Mont-Carlo simulation is carried out to generate 1000 scenarios for gas demand. In order to generate gas demand scenarios using normal PDF, the standard deviation is considered 5\% of gas demand. Other required parameters, which are used in the modeling gas network, are introduced in Appendix.



Figure 4. IEEE 24 bus electricity network interconnected with 15 node gas network.

\subsection{Electricity network description}

The updated version of the IEEE 24-bus reliability test system is used to test the proposed model [43]. Six $200 \mathrm{MW}$-wind farms and two $300 \mathrm{MW}$-electricity storage systems are installed in the network. In the deterministic model, the reserve is considered to be equal to $10 \%$ of the installed capacity of generating units in the deterministic model [36]. A perfect foresight for electricity demand and output wind power of 24 hours is also considered in the optimization. In the stochastic model, however, Mont-Carlo simulation is applied to generate 1000 scenarios of electricity demand and wind power. In order to generate electricity demand scenarios using normal PDF, the standard deviation is considered to be equal to $10 \%$ of electricity demand, and the scale and shape parameters are considered 10 and 200, respectively, to generate wind power scenarios using Weibull PDF [43].

\section{Results analysis}

In this section, the obtained solutions of different strategies of operation with and without taking uncertainty into account, are investigated and analyzed. As mentioned previously, stochastic programming is applied to deal 
with the uncertainty of gas demand, electricity demand, and wind power. For this purpose, 1000 scenarios are generated to present uncertain parameters during the operation period. To reduce the complexity of the model, the generated scenarios are separately reduced and combined. Afterwards, the combined scenarios are reduced again to five scenarios for each uncertain parameter. In this case, this number of scenarios provides an acceptable range of variation for each uncertain parameter. Figure 5 shows the reduced scenarios and their probabilities for this case study.
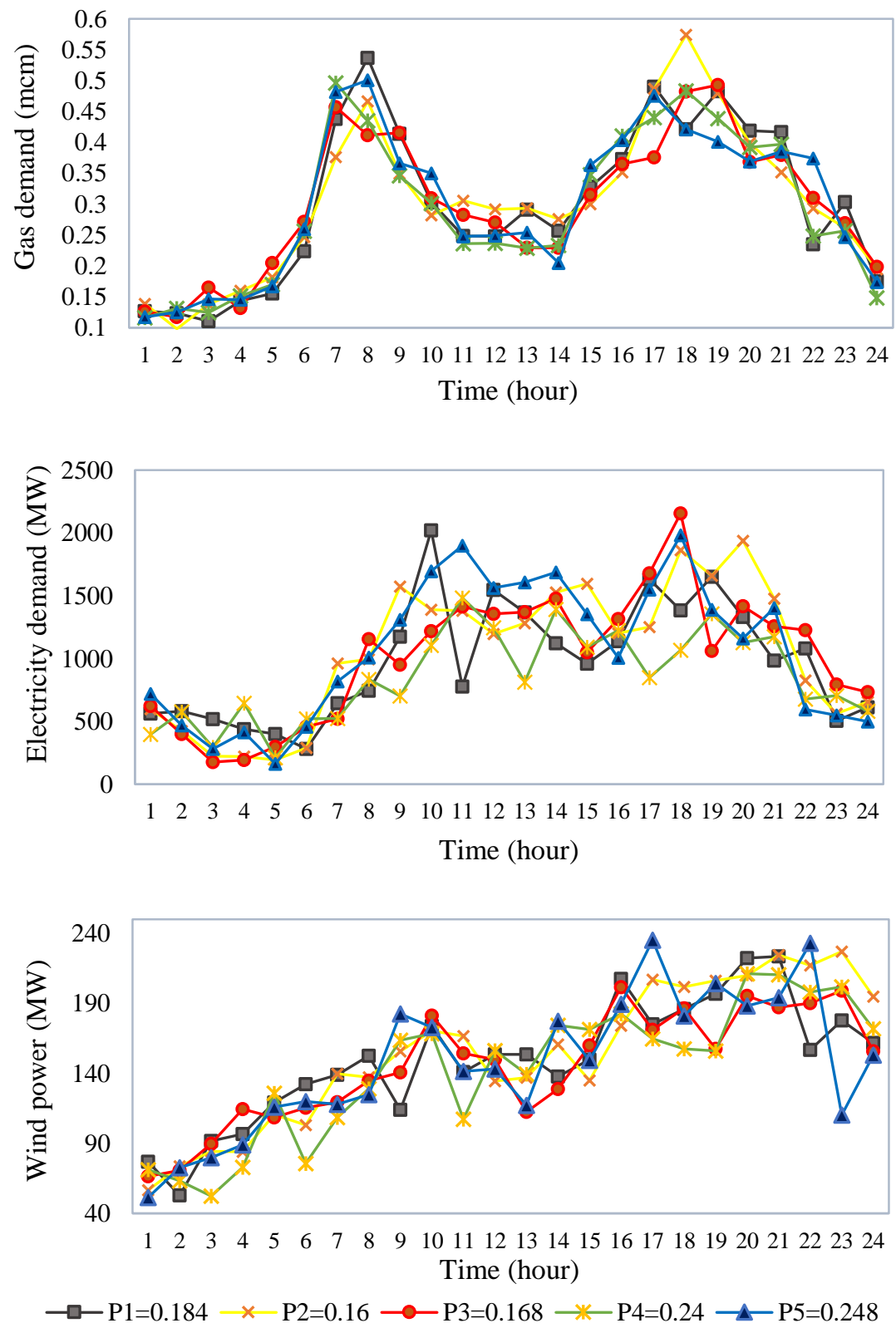

Figure 5. Reduced scenarios for gas demand, electricity demand, and power generation.

\subsection{Computational performance}

This model is solved in Generalized Algebraic Modeling System optimization package (GAMS) via Discrete and Continuous Optimizer solver (DICOPT) [44] using a Core i7 system with $2.67 \mathrm{GHz}$ CPU and 16 GB of RAM. The algorithm in DICOPT is also based on a decomposition method provided in [44]. The decomposition 
method, which is applied to this solver reduces the complexity of the model due to splitting the problem to MILP and Nonlinear Program (NLP) instead of the original MINLP problem [45], and hence obtaining the global optimum is more likely. The deterministic model consists of 2534 equations, 2565 continuous variables, and 2175 binary variables, and the stochastic model consists of 12640 equations, 11935 continuous variables, and 2175 binary variables. The solving time and solution gap for solving the stochastic model as well as the deterministic model through the mentioned operation strategies are demonstrated in Table 3. In Table 3, the number of iterations for each algorithm is presented. As demonstrated, it takes two iterations for solving through the integrated strategy and one iteration for solving through the iterative strategy to successfully converge without slack variables using the proposed algorithm.

Table 3. Solving time and solution gap for coordinated operation of natural gas and electricity networks.

\begin{tabular}{|c|c|c|c|c|}
\hline Modeling & \multicolumn{2}{|c|}{ Stochastic } & \multicolumn{2}{c|}{ Deterministic } \\
\hline Operational strategy & Integrated & Iterative & Integrated & Iterative \\
\hline Solving time (min) & 34.15 & 18.15 & 9.43 & 5.42 \\
\hline Number of iterations & 1 & 2 & 1 & 2 \\
\hline Solution gap (\%) & 0.14 & 0.21 & 0.15 & 0.18 \\
\hline
\end{tabular}

\subsection{Natural gas network operation analysis}

There are considerable oscillations in the gas demand during peak hours (06:00 to 09:00 and 17:00 to 20:00) and off-peak hours (01:00 to 06:00 and 11:00 to 14:00), respectively. The variation of demand impacts the injected gas through the terminal and linepack within the pipelines, which makes the operation of gas networks more challenging.

Figure 6, shows the gas injection through the terminal during the operation period. It is demonstrated that changing the operational strategy to the integrated strategy as well as applying the two-stage stochastic programming, facilitates supplying the demand and prevents high oscillation of gas injection to the network.

Figure 7 depicts the sum of the linepack within pipelines during the operation horizon. It is demonstrated that optimizing the problem through integrated strategy as well as applying stochastic modeling also moderate linepack within the pipelines. In contrast, there are oscillations in the linepack when the deterministic model or iterative operation strategy is applied, which leads to a higher operation of the compressor, and consequently more power is consumed by the compressors. In Fig. 8, the tapped gas of the compressor is presented, in which a more tapped gas leads to an increase in natural gas network operation cost. 


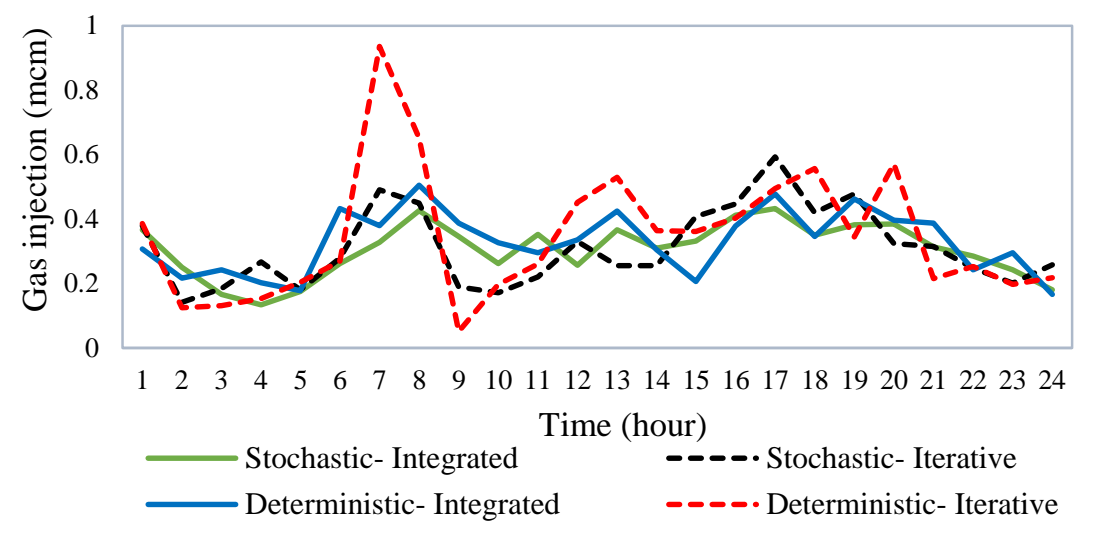

Figure 6. Gas injection of different models.

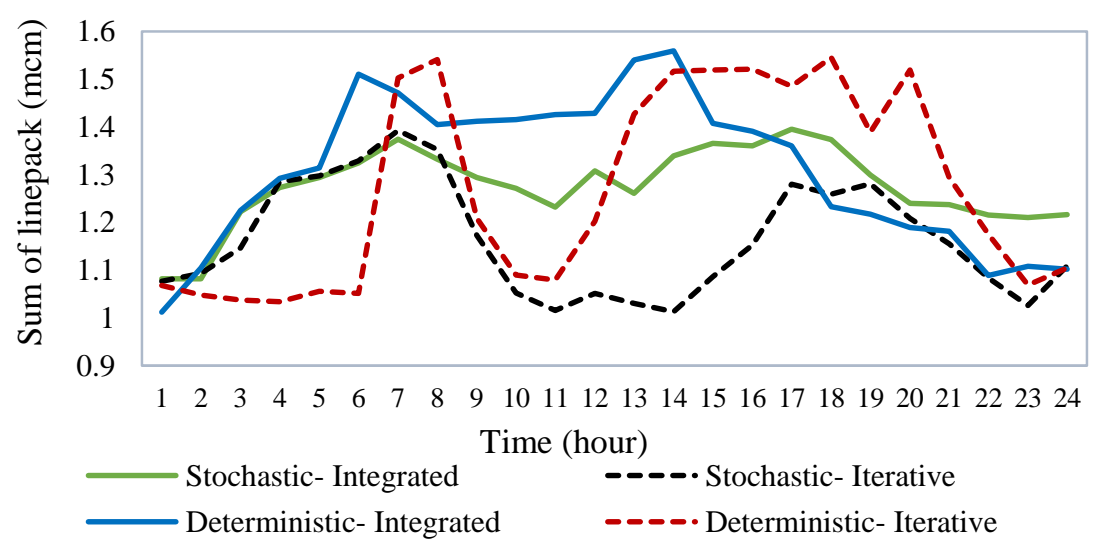

Figure 7. Sum of gas network linepack in different models.
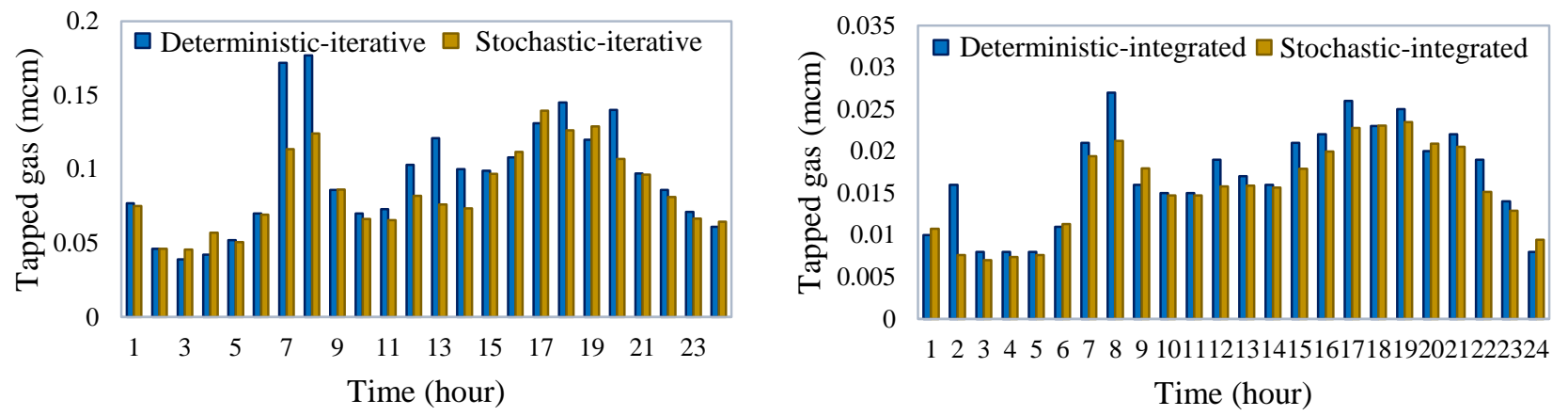

Figure 8. Tapped gas of compressor (expected value is shown in the stochastic model).

\subsection{Electricity network operation analysis}

The output power of different types of power plants during the operation period and the total output power of these power plants are depicted in Fig. 9 and Fig. 10, respectively (the expected values are shown in the stochastic model). In the deterministic model, power plants generated more power to supply the electricity demand against the stochastic model. One reason is that reserve is required in the deterministic model to handle the uncertainty. In order to provide the reserve, nuclear and coal power plants generated more power in the deterministic model compared with the stochastic model. In contrast with the deterministic model, in the stochastic model, wind generators are more operative to meet the demand during the whole period of operation. As a result, the operation cost of the electricity network decreases considering the uncertainty through both operational strategies. Moreover, in stochastic models, charging and discharging of electricity storage systems 
are scheduled more efficiently, which is beneficial to deal with fluctuations in the electricity demand and output power of wind farms.

On the other hand, when the stochastic and deterministic models are optimized through integrated strategy, the gas-fired power plants are almost turned off during off-peak hours of electricity demand and the peak hours of gas demand (from 01:00 to 07:00 and from 19:00 to midnight). However, these power plants are more operative during peak hours of electricity demand, which is due to the advantages of gas-fired power plants, such as providing short startup time and fast ramping rate.

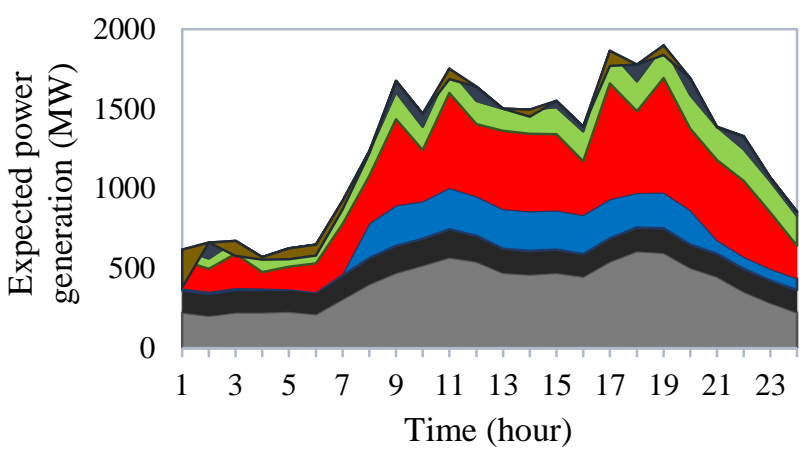

(a) Stochastic under integrated strategy

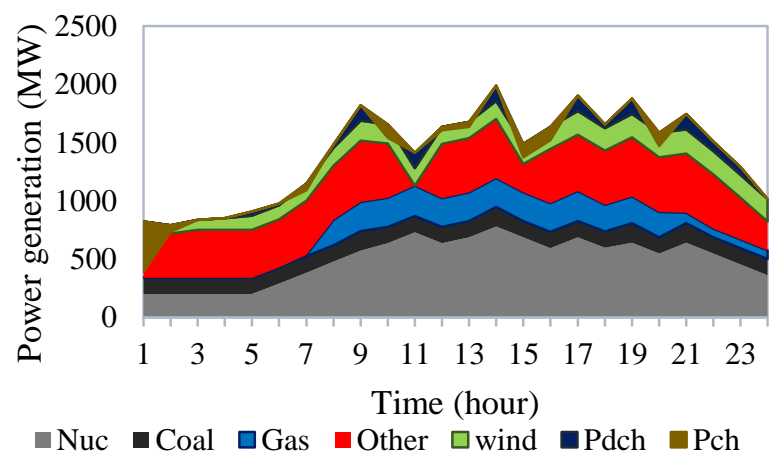

(c) Deterministic under integrated strategy



(b) Stochastic under iterative strategy



(d) Deterministic under iterative strategy

Figure 9. Power generation of each type of power plant during operation period.
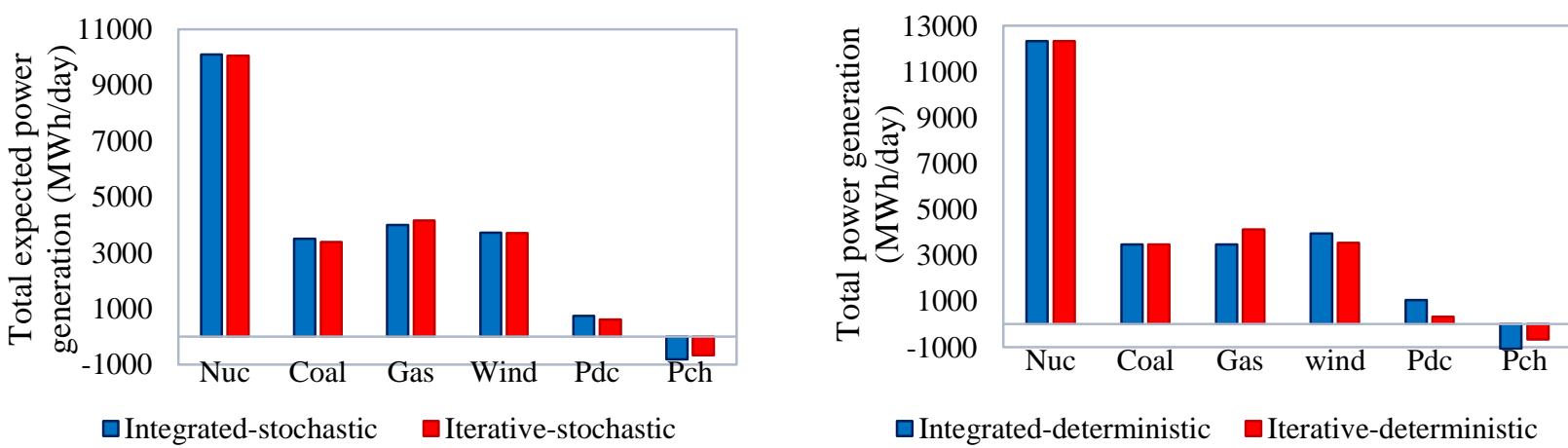

Figure 10. Total power generation by different technologies.

In Fig. 11, the required fuel provided by gas and coal resources to generate electricity during the operation period is depicted. As it is shown, when the models of the natural gas and electricity networks are optimized 
through the integrated strategy, the natural gas consumption of the gas-fired power plants is less than optimizing the problem through the iterative strategy $(0.13 \mathrm{mcm} /$ day and $0.27 \mathrm{mcm} /$ day, respectively). Moreover, in the stochastic model, the coal consumption of the power plants is lower than the deterministic model $(0.11 \mathrm{kton} /$ day and $0.29 \mathrm{kton} /$ day through integrated and iterative strategies, respectively). Therefore, incorporating the uncertainty in this problem and applying the integrated strategy of operation reduce the consumption of fossil fuels, which is beneficial, and consequently, it reduces the GHG emissions (Table 4).

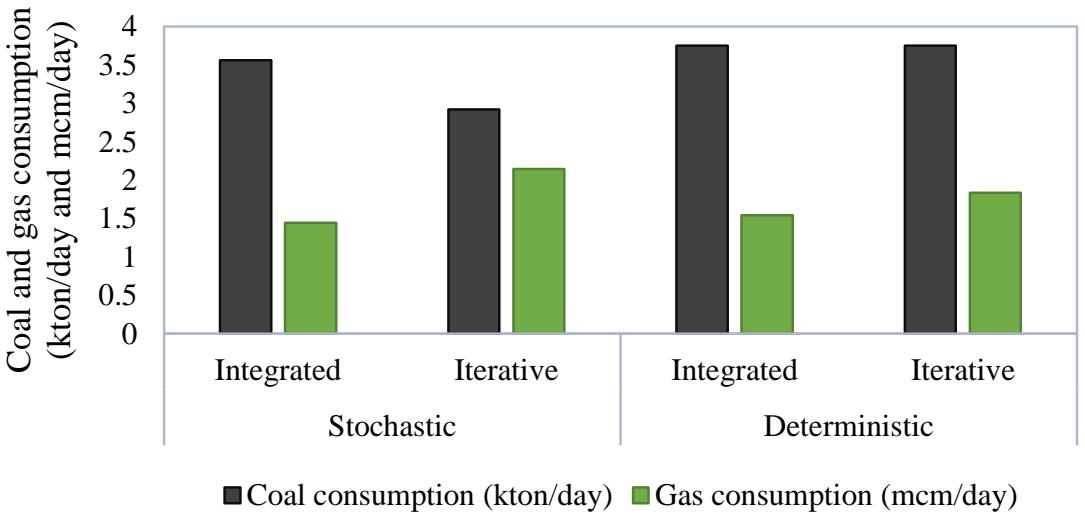

Figure 11. Consumed fossil fuel during the operation period.

Table 4. Produced emission for coordinated operation of natural gas and electricity networks.

\begin{tabular}{|c|c|c|c|c|}
\hline Modeling & \multicolumn{2}{|c|}{ Stochastic } & \multicolumn{2}{c|}{ Deterministic } \\
\hline Operational strategy & Integrated & Iterative & Integrated & Iterative \\
\hline $\begin{array}{c}\text { Produced carbon dioxide by } \\
\text { coal power plants (kton/day) }\end{array}$ & 3.5600 & 2.9224 & 3.7513 & 3.7535 \\
\hline $\begin{array}{c}\text { Produced carbon dioxide by } \\
\text { gas-fired power plants } \\
\text { (kton/day) }\end{array}$ & 1.4466 & 2.1459 & 1.5434 & 1.8369 \\
\hline $\begin{array}{c}\text { Total produced carbon dioxide } \\
\text { (kton/day) }\end{array}$ & 5.0066 & 5.0682 & 5.2947 & 5.5905 \\
\hline
\end{tabular}

According to the obtained results from optimizing the electricity network operation, the expected wind curtailment of the stochastic model and the wind curtailment of the deterministic model are depicted considering both strategies in Fig. 12. The results indicate a lower wind curtailment in the stochastic model compared with the deterministic model, which highlights one of the benefits of stochastic programming. It is shown that most wind curtailment is occurred during off-peak hours of operation. The relatively high amount of wind curtailment in busbars 3,5, and 7 is due to the congestion through the transmission lines as well as the long distances from the electricity storage facilities The main reason for the low wind curtailment in busbars 16, 21, and 23 is due to the short distance from the electricity storage facilities, which bypasses the transmission congestions. 


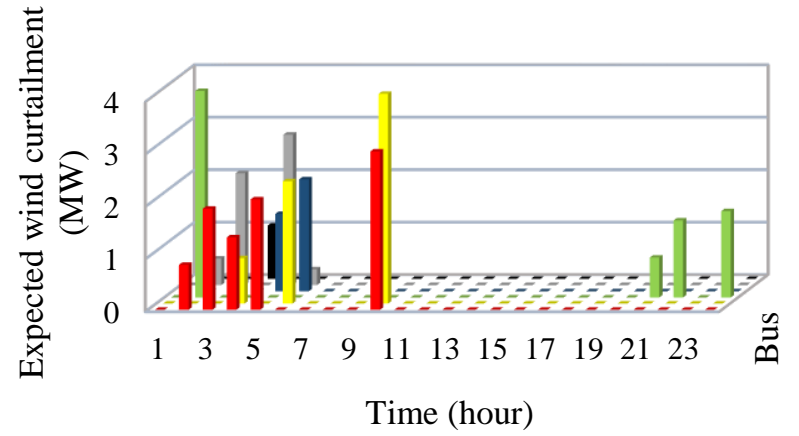

(a) Stochastic under integrated strategy

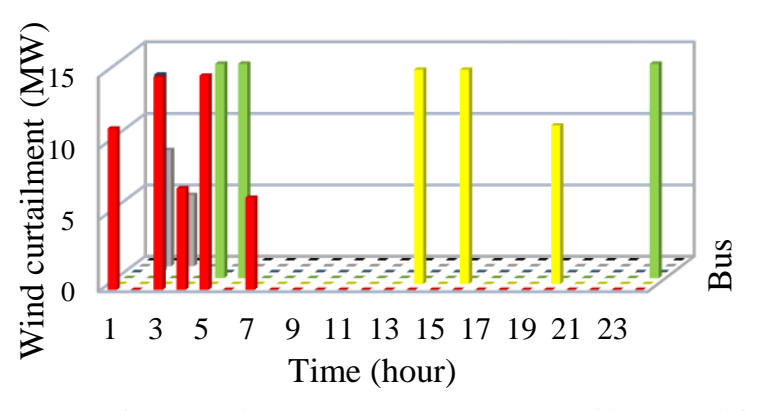

- Bus $3-$ Bus $5 \backsim$ Bus 7 -Bus $16 \backsim$ Bus 21 - Bus 23

(c) Deterministic under iterative stratgey

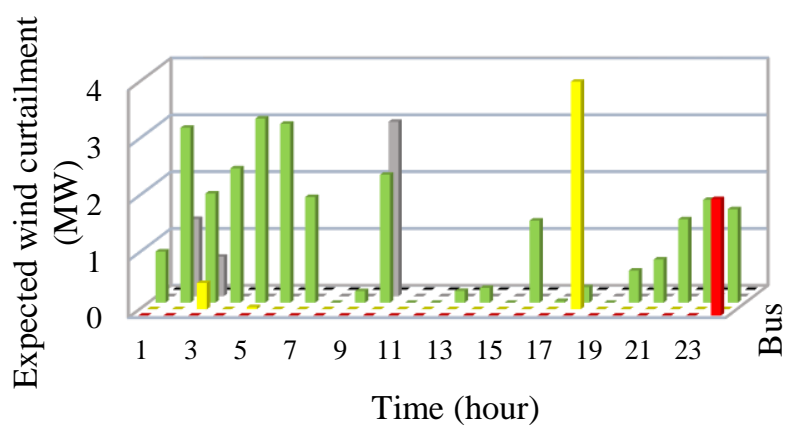

(b) Stochatic under iterative strategy

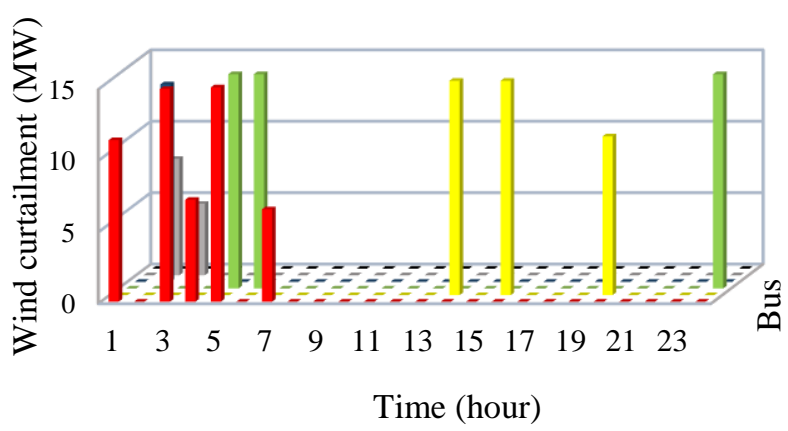

(d) Deterministic under iterative stratgey

Figure 12. Wind curtailment.

In addition to the above advantages of stochastic modeling that leads to cost reduction, there are other benefits in considering uncertainty. For example, it optimizes the charge and discharge of electricity storage systems more efficiently, which increases the lifetime of these systems by preventing unnecessary charging and discharging.

In Fig. 13, the state of charge of the electricity storage systems is depicted, where the state of charge of these systems is minimum, during peak hours, whereas it is maximum during off-peak hours. The standard deviations of the state of charges are lower using the stochastic model (99.62 and 102.81 considering integrated and iterative strategies of operation, respectively), in comparison with applying the deterministic model (148.72 and 102.87 considering integrated and iterative strategies, respectively). The lower standard deviation of the stochastic model shows the better operation of the electricity storage systems that prevents unnecessary charges and discharges and leads to more batteries life span. 


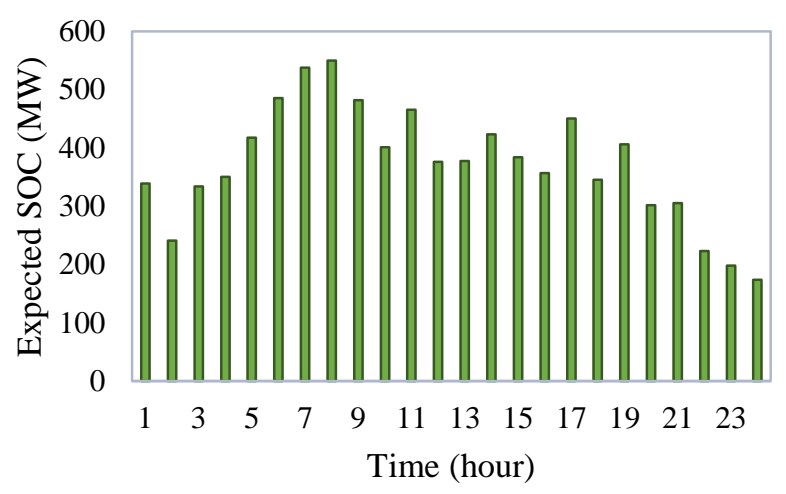

(a) Stochastic model under integrated strategy

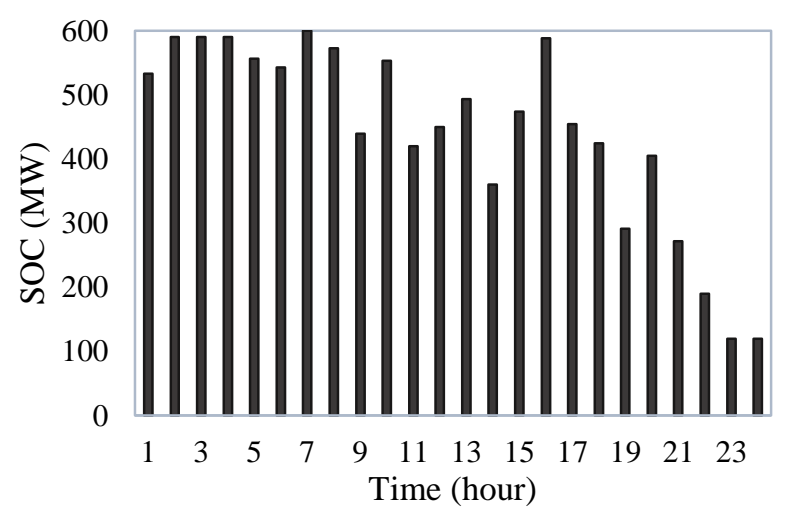

(c) Deterministic model under integrated strategy



(b) Stochastic model under iterative strategy

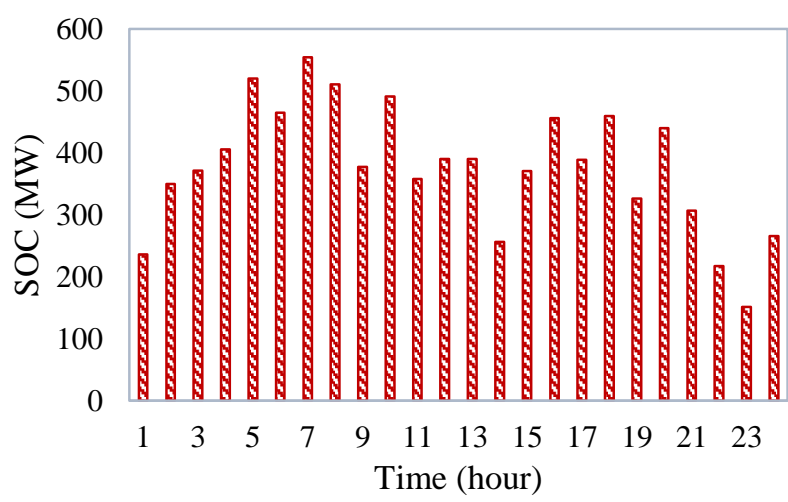

(d) Deterministic model under iterative strategy

Figure 13. State of charge of electricity storage systems.

\subsection{Economic analysis}

The operation cost of stochastic and deterministic models in integrated and iterative strategies are presented in Table 5. According to the obtained results, applying the integrated strategy to optimize the coordinated operation of natural gas and electricity networks reduces the cost of stochastic and deterministic models by $0.1030 \mathrm{~m} /$ day and $0.1696 \mathrm{~m} £ /$ day, respectively. The most important reason is that changing the strategy of operation to integrated leads to a balanced linepack within the pipelines and a balanced gas injection through the terminal. As a result, benefiting from integrated operation strategy, gas network operators can respond more efficiently to the changes in the gas demand and gas requirement of gas-fired generators. Furthermore, applying integrated operational strategy leads to a more efficient charge and discharge of electricity storage systems

Table 5. Operation cost of coordinated operation of gas and electricity networks.

\begin{tabular}{|c|c|c|c|c|}
\hline \multicolumn{2}{|c|}{ Modeling } & \multicolumn{3}{c|}{ Operation cost (m£) } \\
\cline { 3 - 5 } \multicolumn{2}{|c|}{} & $\begin{array}{c}\text { Electricity } \\
\text { network }\end{array}$ & $\begin{array}{c}\text { Natural gas } \\
\text { network }\end{array}$ & Total \\
\hline \multirow{2}{*}{ Stochastic } & Integrated & 0.2618 & 2.4221 & 2.6839 \\
\cline { 2 - 5 } & Iterative & 0.2632 & 2.5237 & 2.7869 \\
\hline \multirow{2}{*}{ Deterministic } & Integrated & 0.2697 & 2.6718 & 2.9415 \\
\cline { 2 - 5 } & Iterative & 0.2798 & 2.8213 & 3.1111 \\
\hline
\end{tabular}

Comparing the results of the coordinated operation of natural gas and electricity networks shows that the stochastic model provides a better solution under different operation strategies. Applying the stochastic model reduces the cost of operation by $0.2576 \mathrm{mf} /$ day and $0.3242 \mathrm{~m} /$ /day through integrated and iterative strategies, 
respectively. The reasons could be mentioned as a more balanced gas supply, a more balanced linepack within the pipelines, no reserve requirements, less operation of the compressor, and a lower wind curtailment.

\subsection{Contingency analysis}

A contingency is defined as a failure of an element, such as a generator in a power system [46]. In practice, the outage of generators leads to an increase in gas demand. This is due to the re-dispatch of gas-fired generators to provide supply-demand balance. In order to examine contingency in this problem, a set of scenarios on generators outages is defined, which leads to load shedding in the electricity network. Then, the amount of occurred load shedding is compared applying the stochastic model and the deterministic model considering both strategies of operation.

Figure 14 indicates the amount of load shedding in the case of contingency. The outage of generators 1, 2, 7, and 8 in which load shedding is occurred are considered as scenarios 1, 2, 3, and 4, respectively. The obtained results of contingency analysis illustrate using the stochastic model reduces the amount of load shedding compared to the deterministic model through iterative and integrated operational strategies. Furthermore, applying the integrated strategy of operation to optimize the coordinated operation of these networks reduces the amount of load shedding. As a result, considering stochastic programming as well as optimizing the problem through the integrated strategy enhances the reliability of the electricity network by reducing energy not supply in the case of contingency. The reason is that the gas-fired power plants are able to deal with variation in supply and demand-side due to a more balanced linepack within the pipelines that provides the possibility to deal with variation without causing congestion in the transmission lines.

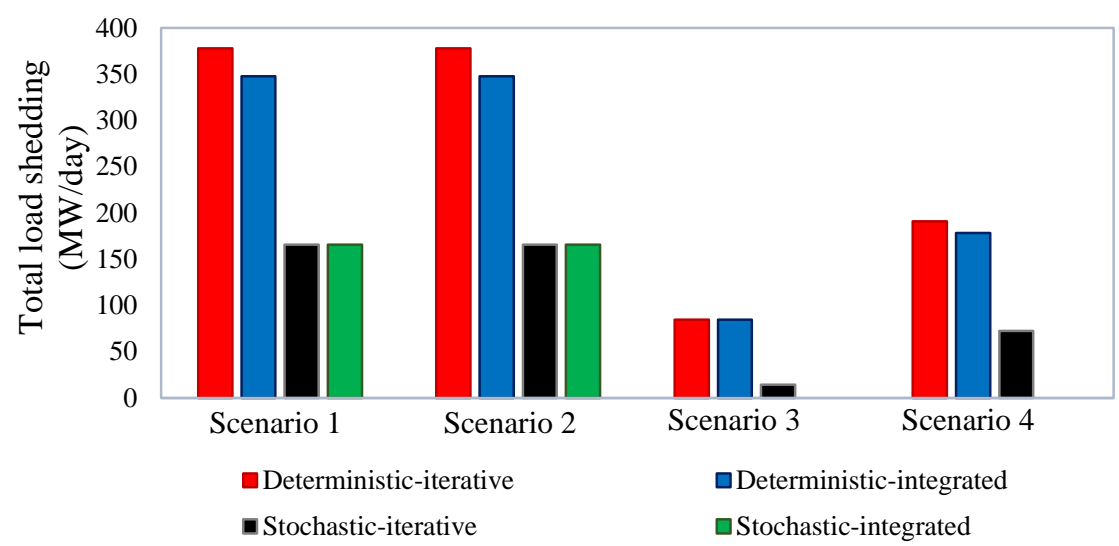

Figure 14. Comparing load shedding in the case of contingency.

\subsection{Value of electricity storage systems in normal and contingency conditions}

In order to evaluate the value of flexibility options, specifically electricity storage, the results are compared with a case that electricity storage is not installed in the system. In Table 6, the operation cost, wind curtailment, and the maximum/minimum of the sum of the linepack with and without electricity storage throughout all the case studies are indicated. This is evident that employing electricity storage systems reduces the cost of electricity and gas networks operation up to $£ 39,400$ during the operation period. In the electricity network, employing these facilities prevents wind curtailment significantly by responding to the changes in the output power of wind farms, which reduces the cost of electricity network operation. On the other hand, the electricity 
storage systems reduce the high peaks and valleys in linepack (up to 45\%), which makes the gas system

426 operation less challenging. It should be noted that reducing the difference between the maximum/minimum sum

427 of linepack by employing these storage systems can prevent unnecessary injection through the terminal, and it

428 can reduce the cost of gas network operation.

Table 6. Comparison of gas and electricity systems operation with and without employing electricity storage systems.

\begin{tabular}{|c|c|c|c|c|c|c|c|c|}
\hline Modeling & \multicolumn{4}{|c|}{ Stochastic } & \multicolumn{4}{c|}{ Deterministic } \\
\hline Operation strategy & \multicolumn{2}{|c|}{ Integrated } & \multicolumn{2}{c|}{ Iterative } & \multicolumn{3}{c|}{ Integrated } & \multicolumn{3}{c|}{ Iterative } \\
\hline $\begin{array}{c}\text { Electricity storage } \\
\text { employment }\end{array}$ & Yes & No & Yes & No & Yes & No & Yes & No \\
\hline $\begin{array}{c}\text { Electricity network } \\
\text { operation cost (mf) }\end{array}$ & 0.2618 & 0.2812 & 0.2632 & 0.2896 & 0.2697 & 0.29022 & 0.2798 & 0.29022 \\
\hline $\begin{array}{c}\text { Gas network } \\
\text { operation cost (mf) }\end{array}$ & 2.4221 & 2.5341 & 2.5237 & 2.6913 & 2.6718 & 2.8403 & 2.8213 & 2.8406 \\
\hline $\begin{array}{c}\text { Wind curtailment } \\
\text { (MWh) }\end{array}$ & 34.78 & 84.01 & 54.92 & 108.11 & 104.23 & 199.61 & 113.73 & 204.21 \\
\hline $\begin{array}{c}\text { Maximum linepack } \\
(\mathrm{mcm})\end{array}$ & 54.92 & 108.11 & 104.23 & 199.61 & 113.73 & 104.21 & 54.92 & 108.11 \\
\hline $\begin{array}{c}\text { Minimum linepack } \\
\text { (mcm) }\end{array}$ & 1.39 & 1.61 & 1.55 & 1.64 & 1.54 & 1.56 & 1.39 & 1.61 \\
\hline
\end{tabular}

On the other hand, as employing electricity storage systems is efficient for dealing with variability and intermittency in the electricity network, the gas-fired power plants can be mostly used in case of contingency, which leads to lower load shedding levels compared to the case that electricity storage is not installed in the system. In Fig. 15, the amount of load shedding reduction compared to the "non-employed storage" case in the contingency condition is presented. In this subsection, the outage of generators 1, 2, 7, and 8 are also assumed as scenarios $1,2,3$, and 4 , respectively.

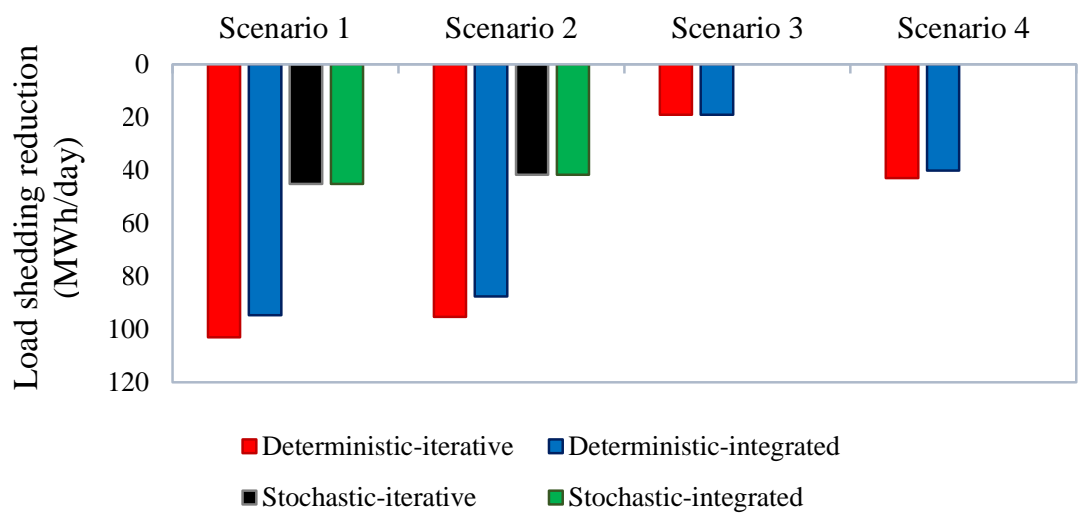

Figure 15. Load shedding reduction compared to "non-employed storage" case.

\section{Conclusion}

Considering the increase in integration of renewable energy resources in the electricity sector, flexible gas-fired power plants could play a crucial role in providing supply-demand balance and couple the electricity and natural gas networks. Therefore, this study examined different aspects of the coordinated operation of these networks. Furthermore, as the role of uncertainty in the coordinated operation of natural gas and electricity networks is 
not deniable, a two-stage stochastic model of these networks was introduced in detail considering the

440 intermittency in the electricity demand, the output power of wind generators, and the non-electric gas demand.

441 In the proposed model, due to the impact of the variability of wind generators on the gas network operation, the

442 role of linepack in meeting the rapid changes in gas demand for power generation was also considered. In order

443 to carry out the stochastic model of these networks, Monte Carlo simulation was applied to generate a number

444 of scenarios representing the gas demand, electricity demand, and wind generation. Then, a backward scenario

445 reduction algorithm was applied based on distances between the scenarios. The models were examined on a 15-

446 node natural gas and IEEE 24 bus electricity network, and stochastic and deterministic models were compared through integrated and iterative operational strategies

According to the results, applying the integrated strategy to optimize the coordinated operation of natural gas and electricity networks reduced the cost of stochastic and deterministic models by $3.83 \%$ and $5.76 \%$, respectively. In addition, it provided some advantages, such as improving gas injection through the terminal, balancing linepack within the pipeline, more efficient charging and discharging of electricity storage systems, and reducing the power consumption by the compressors. Comparing the results of coordinated operation of these networks also demonstrates the advantages of the stochastic model. For example, through applying the stochastic model, the cost of operation was reduced by $9.60 \%$ and $11.63 \%$ in the integrated and iterative strategies. The results also indicate the decrease of wind curtailment in stochastic model compared with the deterministic model. Furthermore, applying stochastic programming also facilitated the gas injection through the terminal and linepack within the pipelines. Besides, defining a set of scenarios on generators outage proved that applying stochastic programming enhances the reliability of the energy system especially when optimizing the operation of natural gas and electricity networks through the integrated operational strategy. Furthermore, the role of electricity storage systems was quantified in the case of normal and contingency conditions. The results show the benefits of these systems, such as operation cost reduction, wind curtailment reduction, and load shedding decrement in the case of contingency.

As future research, applying an approach that is not based on scenario is suggested to cope with the uncertainty of this problem, such as possibilistic programming or robust programming in which there is no need to consider a number of scenarios. This is due to the complexity of this problem, which is a mixed-integer nonlinear problem, and adding a number of scenarios increases the complexity of the problem and the solving time considerably. However, if it is necessary to deal with uncertainty applying a scenario-based approach, using a decomposition technique, such as Benders decomposition, could be beneficial to solve this problem.

\section{Acknowledgment}

470 The authors gratefully acknowledge the "SHAR-LLM" project which has received funding from the European 471 Union's Horizon 2020 research and innovation program under award number 691895 as well as the EPSRC472 funded program "Integrated Development of Low-Carbon Energy Systems (IDLES)" under award number 473 EP/R045518/1. 


\section{Appendix. Gas network data}

The costs of gas supply and gas shedding are considered $0.35 £ / \mathrm{cm}$ and $11.1 £ / \mathrm{cm}$, respectively. The gas demand profile is presented in Fig. 16 and Table 7 [37]. The pipeline data is also presented in Table 8.

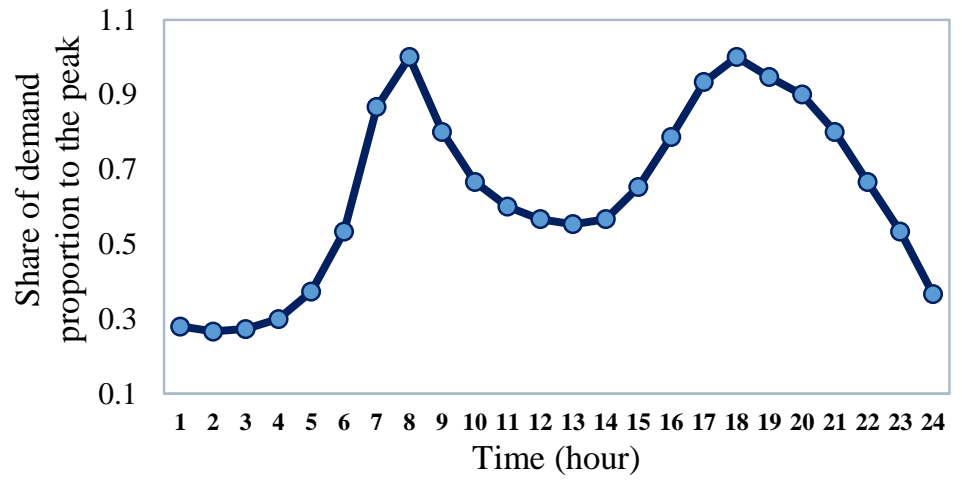

Figure 16. Gas demand (non-electrical)

Table 7. Gas peak of each node.

\begin{tabular}{|c|c|c|c|c|c|}
\hline Node & Gas peak $(\mathrm{cm})$ & Node & Gas peak $(\mathrm{cm})$ & Node & Gas peak $(\mathrm{cm})$ \\
\hline 1 & 550000 & 6 & 400000 & 11 & 400000 \\
\hline 2 & 550000 & 7 & 550000 & 12 & 400000 \\
\hline 3 & 550000 & 8 & 550000 & 13 & 400000 \\
\hline 4 & 550000 & 9 & 550000 & 14 & 400000 \\
\hline 5 & 550000 & 10 & 400000 & 15 & 400000 \\
\hline
\end{tabular}

Table 8. Pipeline data.

\begin{tabular}{|c|c|c|c|c|c|c|c|c|c|}
\hline $\begin{array}{c}\text { Pipe } \\
\text { number }\end{array}$ & $\begin{array}{c}\text { From } \\
\text { node }\end{array}$ & $\begin{array}{c}\text { To } \\
\text { node }\end{array}$ & $\begin{array}{c}\text { Length } \\
(\mathrm{m})\end{array}$ & $\begin{array}{c}\text { Diameter } \\
(\mathrm{mm})\end{array}$ & $\begin{array}{c}\text { Pipe } \\
\text { number }\end{array}$ & $\begin{array}{c}\text { From } \\
\text { node }\end{array}$ & $\begin{array}{c}\text { To } \\
\text { node }\end{array}$ & $\begin{array}{c}\text { Length } \\
(\mathrm{m})\end{array}$ & $\begin{array}{c}\text { Diameter } \\
(\mathrm{mm})\end{array}$ \\
\hline 1 & 1 & 2 & 22211 & 157 & 9 & 7 & 8 & 81 & 43 \\
\hline 2 & 1 & 3 & 24035 & 590 & 10 & 8 & 9 & 6563 & 888 \\
\hline 3 & 1 & 4 & 5585 & 438 & 11 & 6 & 10 & 7636 & 309 \\
\hline 4 & 4 & 3 & 16322 & 438 & 12 & 10 & 11 & 3917 & 309 \\
\hline 5 & 3 & 5 & 6952 & 438 & 13 & 10 & 12 & 97 & 309 \\
\hline 6 & 5 & 6 & 4287 & 309 & 14 & 12 & 13 & 10123 & 590 \\
\hline 7 & 5 & 7 & 4439 & 438 & 15 & 11 & 14 & 5520 & 157 \\
\hline 8 & 5 & 8 & 5032 & 304 & 16 & 11 & 15 & 4298 & 309 \\
\hline
\end{tabular}

\section{References}

[1]. Zantye, M. S., Arora, A., \& Hasan, M. F. (2019). Operational power plant scheduling with flexible carbon capture: A multistage stochastic optimization approach. Computers \& Chemical Engineering, 130, 106544.

[2]. United Nations Framework Convention on Climate Change, Paris Agreement- status of ratification; 5 October 2016. 〈http://unfccc.int/paris_agreement/items/9444.php〉 [Accessed 15 December 2016].

[3]. Hörnlein, L. (2019). The value of gas-fired power plants in markets with high shares of renewable energy: A real options application. Energy Economics.

[4]. Shivaie, M., Ameli, M. T., Sepasian, M. S., Weinsier, P. D., \& Vahidinasab, V. (2015). A multistage framework for reliability-based distribution expansion planning considering distributed generations by a self-adaptive global-based harmony search algorithm. Reliability Engineering \& System Safety, 139, 68-81.

[5]. Su, H., Zio, E., Zhang, J., Li, X., Chi, L., Fan, L., \& Zhang, Z. (2019). A method for the multi-objective optimization of the operation of natural gas pipeline networks considering supply reliability and operation efficiency. Computers \& Chemical Engineering, 131, 106584. 
[6]. Alvarez, G. E. (2019). Integrated scheduling from a diversity of sources applied to the Argentine Electric Power and Natural Gas Systems. Computers \& Chemical Engineering, 106691.

[7]. He, C., Zhang, X., Liu, T., Wu, L., \& Shahidehpour, M. (2018). Coordination of Interdependent Electricity Grid and Natural Gas Network-a Review. Current Sustainable/Renewable Energy Reports, 5(1), 23-36. [6].

[8]. Ameli, H., Qadrdan, M., \& Strbac, G. (2017). Value of gas network infrastructure flexibility in supporting cost effective operation of power systems. Applied energy, 202, 571-580.

[9]. Liu, C., Shahidehpour, M., \& Wang, J. (2011). Coordinated scheduling of electricity and natural gas infrastructures with a transient model for natural gas flow. Chaos: An Interdisciplinary Journal of Nonlinear Science, 21 (2), 025102.

[10]. Qadrdan, M., Wu, J., Jenkins, N., \& Ekanayake, J. (2014). Operating strategies for a GB integrated gas and electricity network considering the uncertainty in wind power forecasts. IEEE Transactions on Sustainable Energy, 5(1), $128-138$.

[11]. Alabdulwahab, A., Abusorrah, A., Zhang, X., \& Shahidehpour, M. (2017). Stochastic security-constrained scheduling of coordinated electricity and natural gas infrastructures. IEEE Systems Journal, 11(3), 1674-1683.

[12]. Zhang, X., Shahidehpour, M., Alabdulwahab, A., \& Abusorrah, A. (2016). Hourly electricity demand response in the stochastic day-ahead scheduling of coordinated electricity and natural gas networks. IEEE Transactions on Power Systems, 31(1), 592-601.

[13]. He, C., Wu, L., Liu, T., \& Shahidehpour, M. (2017). Robust co-optimization scheduling of electricity and natural gas systems via ADMM. IEEE Transactions on Sustainable Energy, 8(2), 658-670.

[14]. Chaudry, M., Jenkins, N., \& Strbac, G. (2008). Multi-time period combined gas and electricity network optimisation. Electric power systems Research, 78(7), 1265-1279.

[15]. Devlin, J., Li, K., Higgins, P., \& Foley, A. (2017). A multi vector energy analysis for interconnected power and gas systems. Applied energy, 192, 315-328.

[16]. Qadrdan, M., Ameli, H., Strbac, G., \& Jenkins, N. (2017). Efficacy of options to address balancing challenges: Integrated gas and electricity perspectives. Applied energy, 190, 181-190.

[17]. Sirvent, M., Kanelakis, N., GEIßLER, B., \& Biskas, P. (2017). Linearized model for optimization of coupled electricity and natural gas systems. Journal of Modern Power Systems and Clean Energy, 5(3), 364-374.

[18]. Manshadi, S. D., \& Khodayar, M. E. (2018). Coordinated operation of electricity and natural gas systems: A convex relaxation approach. IEEE Transactions on Smart Grid.

[19]. Sayed, A. R., Wang, C., Bi, T., \& Masood, A. (2018). A Tight MISOCP Formulation for the Integrated Electric-Gas System Scheduling Problem. Paper presented at the 2018 2nd IEEE Conference on Energy Internet and Energy System Integration (EI2).

[20]. Ameli, H., Qadrdan, M., \& Strbac, G. (2019). Coordinated operation strategies for natural gas and power systems in presence of gas-related flexibilities. IET Energy Systems Integration, 1(1), 3-13.

[21]. Bai, L., Li, F., Cui, H., Jiang, T., Sun, H., \& Zhu, J. (2016). Interval optimization based operating strategy for gaselectricity integrated energy systems considering demand response and wind uncertainty. Applied energy, 167, $270-279$.

[22]. Chuan, H., Tianqi, L., Lei, W., \& Shahidehpour, M. (2017). Robust coordination of interdependent electricity and natural gas systems in day-ahead scheduling for facilitating volatile renewable generations via power-to-gas technology. Journal of Modern Power Systems and Clean Energy, 5(3), 375-388.

[23]. Yuan, H., Haoran, L., Zhaohong, B., \& Baorong, Z. (2017). Unified probabilistic gas and power flow. Journal of Modern Power Systems and Clean Energy, 5(3), 400-411.

[24]. Gazijahani, F. S., Ravadanegh, S. N., \& Salehi, J. (2018). Stochastic multi-objective model for optimal energy exchange optimization of networked microgrids with presence of renewable generation under risk-based strategies. ISA transactions, 73, 100-111.

[25]. Villanueva, D., Feijóo, A. E., \& Pazos, J. L. (2014). An analytical method to solve the probabilistic load flow considering load demand correlation using the DC load flow. Electric power systems Research, 110, 1-8. 
[26]. Biswas, P. P., Suganthan, P., Mallipeddi, R., \& Amaratunga, G. A. (2019). Optimal reactive power dispatch with uncertainties in load demand and renewable energy sources adopting scenario-based approach. Applied Soft Computing, 75, 616-632.

[27]. Mohseni-Bonab, S. M., \& Rabiee, A. (2017). Optimal reactive power dispatch: a review, and a new stochastic voltage stability constrained multi-objective model at the presence of uncertain wind power generation. IET Generation, Transmission \& Distribution, 11(4), 815-829.

[28]. Römisch, W. (2009). Scenario reduction techniques in stochastic programming. Paper presented at the International Symposium on Stochastic Algorithms.

[29]. Razali, N. M., \& Hashim, A. (2010). Backward reduction application for minimizing wind power scenarios in stochastic programming. Paper presented at the 2010 4th International Power Engineering and Optimization Conference (PEOCO).

[30]. Dupačová, J., Consigli, G., \& Wallace, S. W. (2000). Scenarios for multistage stochastic programs. Annals of Operations Research, 100(1-4), 25-53.

[31]. Growe-Kuska, N., Heitsch, H., \& Romisch, W. (2003). Scenario reduction and scenario tree construction for power management problems. Paper presented at the 2003 IEEE Bologna Power Tech Conference Proceedings.

[32]. Davis, P. K., \& Popper, S. W. (2019). Uncertainty Analysis to Better Confront Model Uncertainty: RAND.

[33]. Conejo, A. J., Carrión, M., \& Morales, J. M. (2010). Decision making under uncertainty in electricity markets (Vol. 1): Springer.

[34]. Osiadacz, A. J., \& centre, C. s. (1987). Simulation and analysis of gas networks.

[35]. Ameli, H., Qadrdan, M., Strbac, G., \& Ameli, M. T. (2020). Investing in Flexibility in an Integrated Planning of Natural Gas and Power Systems. IET Energy Systems Integration, 2(2), 101-111.

[36]. Ahmadi, A., Nezhad, A. E., \& Hredzak, B. (2019). Security-constrained unit commitment in presence of lithium-ion battery storage units using information-gap decision theory. IEEE Transactions on Industrial Informatics, 15(1), 148-157.

[37]. Burer, S., \& Letchford, A. N. (2012). Non-convex mixed-integer nonlinear programming: A survey. Surveys in Operations Research and Management Science, 17(2), 97-106.

[38]. Köppe, M. (2012). On the complexity of nonlinear mixed-integer optimization. In Mixed Integer Nonlinear Programming (pp. 533-557): Springer.

[39]. Qadrdan, M., Abeysekera, M., Wu, J., Jenkins, N., \& Winter, B. (2020). The Future of Gas Networks. In The Future of Gas Networks (pp. 49-68): Springer.

[40]. Chaudry, M., Wu, J., \& Jenkins, N. (2013). A sequential Monte Carlo model of the combined GB gas and electricity network. Energy Policy, 62, 473-483.

[41]. G. Strbac, D. Pudjianto, R. Sansom, P. Djapic, H. Ameli, N. Shah, N. Brandon, A. Hawkes, and M. Qadrdan, "Analysis of Alternative UK Heat Decarbonisation Pathways for the Committee on Climate Change", Imperial College, London, Aug. 2018.

[42]. Ordoudis, C., Pinson, P., González, J. M. M., \& Zugno, M. (2016). An Updated Version of the IEEE RTS 24-Bus System for Electricity Market and Power System Operation Studies.

[43]. Chang, T. P. (2010). Investigation on frequency distribution of global radiation using different probability density functions. International Journal of Applied Science and Engineering, 8(2), 99-107.

[44]. 'Generalized Algabric Modeling System' (https://www.gams.com/latest/docs/S_DICOPT.html).

[45] Ameli, H., Qadrdan, M., \& Strbac, G. (2020). Coordinated Operation of Gas and Electricity Systems for Flexibility Study", Frontiers in Energy Research, 8, 120.

[46]. Jamei, M., Schweitzer, E., Scaglione, A., \& Hedman, K. W. (2018). Gas and electric grid unit commitment with coordinated N-1 generator contingency analysis. Paper presented at the 2018 Power Systems Computation Conference (PSCC). 